\title{
Evaluating the impacts of school garden-based programmes on diet and nutrition-related knowledge, attitudes and practices among the school children: a systematic review
}

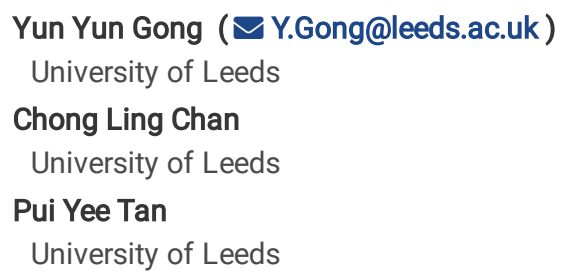

\section{Research Article}

Keywords: school-aged children, school garden-based programmes, nutritional knowledge, attitudes, food acceptability, dietary practices, fruits and vegetables

Posted Date: February 25th, 2022

DOI: https://doi.org/10.21203/rs.3.rs-1359057/v1

License: (c) (i) This work is licensed under a Creative Commons Attribution 4.0 International License. Read Full License 


\section{Abstract}

Background: Previous evidence suggests that school garden-based programmes (SGBP) may be a promising yet cost-effective intervention to improve children's knowledge, attitudes and practices (KAP) on healthy eating. This review aimed to summarise and evaluate the evidence available on the impacts of SGBP in addressing diet and nutrition-related KAP among school-aged children.

Methods: 5 databases including PubMed, Embase, Cochrane, Web of Science and Scopus were searched until February 2021. Randomised, nonrandomised controlled and pre-post intervention studies investigating the impacts of SGBP on at least one of the outcomes of interest including diet and nutrition-related knowledge, attitudes towards fruits and vegetables (F\&V), food diversity and dietary practice among school-aged children were included. Study selection and data extraction were performed by one reviewer and checked for accuracy by the other two reviewers in accordance with PRISMA guideline. Quality appraisal for studies included was assessed using American Dietetic Association Quality Criteria Checklist.

Results: A total of 10,836 records were identified, and 35 studies that met the inclusion and exclusion criteria were included. This includes 25,726 students from 341 schools and 8 nurseries from 12 countries. Intervention duration ranged from 6 weeks to 4 years with 18 studies involving a varied degree of parental participation. SGBP, which majorly includes school gardening activities, cooking lessons and nutrition education, demonstrated beneficial effects on children nutritional knowledge, and their attitudes and acceptability towards vegetables, but not for fruits. However, no strong evidence supports its positive impact on children's dietary practices including the actual F\&V consumption and dietary diversity. No significant impact of parental involvement was found on the measured outcomes which may be partly due to poor parental engagement or low activity intensity during the intervention.

Conclusion: These findings suggest that SGBP may be effective in promoting children's nutritional knowledge, attitudes and acceptability towards vegetables, however, the impacts may vary by the type, the extent, and the length of the programmes, and other factors such as parent involvement. Future SGBP is suggested to implement using a combined multidisciplinary approach targeting the children, parents, and community to effectively promote healthy eating among the children and prevent childhood obesity.

\section{Introduction}

Childhood malnutrition in all forms affects every country in the world [1]. In the past four decades, a tenfold increase was reported in the number of obese children and adolescents aged 5 to 19 worldwide, from 11 million in 1975 to 124 million in 2016 with an addition of 213 million being classified as overweight [2]. Whereas 50 and 150 million children under the age of 5 were reported to be wasted and stunted, respectively [3]. Childhood malnutrition may lead to severe health consequences for physical growth and cognitive development. Concerningly, childhood malnutrition is likely to persist into adulthood, which can perpetuate an ill-health cycle, increasing the health risk in their later life [4].

Suboptimal diet with poor dietary behaviour is one of the major contributing factors to malnutrition. A healthy diet, according to the World Cancer Research Fund [5] and WHO [6], is characterised by the consumption of abundant whole grains, legumes, fruits, vegetables, and nuts with a limited intake of salt, red and processed meat, sugar and fat-rich "fast food" and other processed food. Diet rich in fibre and fruits and vegetables (F\&V) e.g. Mediterranean diet, has shown positive effects on tackling obesity [7-11]. Despite the prominent benefits of F\&V, the current consumption level remains low in young people. A survey of ten European countries reported that only $23.5 \%$ of the studied children met the WHO [14] requirement of no less than 400 grams of F\&V per day and more than half of the children do not consume fruits on a daily basis [12].

According to the PRECEED-PROCEED model, behavioural change occurs under the changes of its determinants [13]. In other words, having a deeper understanding of its underlying determinant is the first step in improving diet quality among children. Compelling evidence suggested that F\&V consumption is driven by knowledge and awareness of, preference for and attitude towards such foods [14]. Food preferences and dietary habits are generally shaped at an early age, and they are more likely to persist into adulthood and affect our food choices in later life. Therefore, there is a need to enhance nutritional knowledge and encourage early F\&V exposure among the children, to promote their willingness to consume, acceptance and liking of F\&V [15-19].

Recent evidence suggested that school garden-based programmes (SGBP) may be a promising yet cost-effective intervention to promote healthy eating habits and increase children's F\&V intake [20]. School is regarded as a prime setting to shape children's dietary behaviour whereby $20 \%$ of their daily dietary intake are obtained [21,22]. SGBP, which enhance the circular learning environment by integrating a hands-on experimental approach, may strengthen the impact of nutrition education on children. The hands-on activities include direct gardening experiences and active involvement in designing, building, developing and maintaining the school garden with edible plants [19,23]. Other activities may include bed preparation, seed planting, seedlings transplanting, plant growing and nurturing, and application of organic pest control [19]. Growing own produces not only can increase school and/or home accessibility and availability of $F \& V$, but also encourage children to appreciate and value garden produce $[24,25]$. This may eventually increase children's preferential selection, willingness to taste and potentially the intake of F\&V. In addition to singlecomponent SGBP intervention, multicomponent school garden-based interventions that integrate gardening with classroom curriculum, physical education, cooking session, food service, and/or with parental involvement displayed a more promising effect in promoting children's F\&V consumption and its determinants $[21,23,26]$. 
Despite greater potential evidence on SGBP effects towards improving knowledge, attitudes and practises (KAP) regarding diet and nutrition remain mixed. Therefore, this study aimed to systematically review the available evidence on the impacts of SGBP on diet and nutrition-related KAP among school-aged children, and to explore the key features of its effectiveness.

\section{Methods \\ Search strategy}

The search was conducted between $11^{\text {th }}$ November 2020 to $6^{\text {th }}$ February 2021.5 databases were used, including PubMed, Embase, Cochrane, Web of Science and Scopus for primary research articles published from year 2000. This timeframe was chosen with the aim of obtaining the most recent SGBP intervention studies. The following search terms were used: (1) school children as the targeted population: adolescent* OR boy? OR child* OR children OR girl? OR juvenil* OR kid? OR preschool* OR school* OR teen* OR youth* OR young OR "school children" OR student*; (2) school setting: school* OR nurser* OR kindergarten* OR kindergarden*; (3) garden-based interventions: garden* OR gardening OR plant* OR fruit* OR vegetable* OR "fruit vegetable*" OR "fruit growing" OR "vegetable growing" OR seed* OR tree* OR "organic agriculture" OR "organic farming" OR "organic food" OR farm; (4) outcome measures on diet and nutritional related KAP: (eating OR diet* OR food OR dietary OR nutrition OR nutritional OR fruit* OR vegetable*) AND (knowledge OR attitude OR practi? $e^{\star}$ OR behavio? r^ OR preference* OR habit* OR intake* OR consumption* OR healthy OR skill* OR pattern* OR diversity OR diverse OR perception*) OR "energy intake" OR "appetite" OR "portion size*" OR "food fussiness" OR "food neophobia"; (5) study design: "controlled trial*” OR "intervention" OR randomised OR randomized OR trial* OR "randomised controlled trial*" OR "randomized controlled trial*" OR follow-up stud* OR program evaluation*" OR "controlled before-after stud*”. Details of the search strategies used for each database are presented in the Supplementary Table 1.

\section{Inclusion and exclusion criteria}

Studies were included if they met the following inclusion and exclusion criteria.

\section{Inclusion criteria:}

Population: School children and adolescents (boys and girls) aged 3-18 years old attending nursery, kindergarten, primary, secondary or high school education and special school. Children under the age of 3 and over the age of 18 would still be included as long as they were being classified as "students" or still attending nurseries, kindergarten or high schools.

Interventions: Studies that used school gardening, kitchen-gardening, garden curriculum or horticulture activities as primary interventions were included. Gardening activities included cultivating plants such as fruits, vegetables, shrubs, flowers and trees while gardening programmes included activities such as preparing the soil, sowing seeds, planting, weeding, watering and harvesting, hands-on learning with fruits and vegetables, education on food origins and systems, and the fresh produce's production. Garden-related cooking and tasting activities were also included. Gardening programmes could be conducted within the school curriculum or conducted out of the lesson time such as during recess, lunchtime or after-school activities and school trips to community allotments.

Outcomes: Studies with a result for at least one outcome of interest were included, including examining food literacy such as diet and nutritionrelated knowledge, attitudes, skills, preferences, behaviours and practices e.g. dietary diversity and F\&V intake.

Study design: Randomised controlled trials in which individuals or clusters (classes or schools) were randomly assigned to trial arms, nonrandomized controlled trials and pre-post intervention studies which examined the changes in the outcome measures at post-intervention and baseline were included. Only studies written in English were included. No restrictions were placed on the author, sample size, funding sources of study, duration of the intervention or the country where the intervention took place.

\section{Exclusion criteria}

Garden-based interventions that did not organise by the school such as community-based gardening programmes, community youth interventions, summer holiday extra-curricular activities or clubs were excluded. Study organised by the school but occurred at the community level such community gardens, however, were included as the participants were still being regarded as "students". Interventions with only teaching gardening related knowledge without actual hand-on gardening component were excluded. Studies that did not regard school gardening as their primary intervention or did not specify the age of participants were also excluded. Studies that only focused on describing school-based gardening programme without addressing its effects on nutritional KAP were excluded. Editorials, commentaries, opinions, review articles and observational studies such as cross-sectional studies, prospective and retrospective cohort studies were not included as well as unpublished, grey literature and ongoing studies with only preliminary findings.

Page $3 / 34$ 


\section{Study selection}

Studies obtained from the search were uploaded to ENDNOTE (X7, Thomson Reuters). Screening and selection of studies for inclusion in this review were performed by a reviewer and the decisions were checked by the other reviewer. During the first round of screening, the title and abstract were checked for eligibility based on the inclusion and exclusion criteria. In the second round of screening, full-text articles were obtained and screened for eligibility using the same criteria. Disagreement between reviewers was resolved by discussion and by a third reviewer.

\section{Data extraction}

A standardized data extraction form was utilized to obtain the following information, where possible: author, year of publication, journal source, source of funding, study design, year of study, country or population, sample characteristics (e.g. gender, age, socioeconomic status etc.), sample size, intervention size, control size, intervention group description (activities included in the SGBP), control group description, duration of intervention, outcome measures (e.g. indicators related to KAP around diet and nutrition) and main findings. Any disagreements were resolved through discussion with the research team.

\section{Strategy for data synthesis}

A systematic review synthesising the qualitative evidence of school garden-based programmes was conducted. The findings on the impact of school garden-based programmes in affecting school children's KAP around diet and nutrition were reported according to the components of the interventions via categorising them into school garden-based programmes with and without parental involvement. A meta-analysis on any of the quantitative data extracted was unable to be performed due to the heterogeneity and variation in the study design, outcome measurement and intervention component.

\section{Risk of bias assessment}

The risk of bias of the individual studies included was independently assessed by two reviewers. Any disagreement on the risk of bias between reviewers was resolved by discussion and by a third reviewer when necessary. The risk of bias of the individual studies included was assessed using the Academy of Nutrition and Dietetics, Quality Criteria Checklist [27]. The 10 questions focus on (1) how clear the research question was; (2) selection of participants; (3) randomization/ group comparability; (4) description of withdrawals; (5) how the blinding was; (6) whether study procedures were described clearly; (7) whether the outcomes were clearly defined; (8) were appropriate statistical analyses applied; (9) did the results support the conclusion; (10) funding or sponsorship bias. To be rated positive, each of the criteria 2, 3, 6 and 7 must be met and the majority of 10 criteria overall. Any of criteria 2, 3, 6 and 7 not being met resulted in a neutral rating. If most criteria (i.e., more than 6 of them) were not met, the article would have a negative rating.

\section{Results}

The search from literature yielded a total of 10,836 potentially relevant articles from 5 databases (Fig. 1). After removing duplicates, 4914 records remained. Those articles were screened for title and abstract for eligibility, resulting in 4737 records being excluded. The full text of the remaining 177 records was assessed and examined. Using the same criteria, a total of 141 records were excluded. Thus, a total of 35 records were included in this review.

\section{Study Characteristics}

In total, 25,726 school children recruited from 341 schools and 8 nursery centres from 12 different countries were included in this review. Most of the studies were mainly reported from the populations of the United States $(n=18)$. Six studies were conducted in developing countries (Nepal, Bhutan, Burkina Faso and Brazil) and 29 studies were conducted in developed countries (United States, Australia, United Kingdom, Portugal, Canada, South Korea, Netherlands and Belgium). Participants' age ranged from 2 to 19 years old, with the majority from the age of $8-12$ years old. Duration of intervention ranged from 6 weeks to 4 years (mean \pm SD: $10 \pm 11$ months) and integrated school gardening intervention activities included outdoor or indoor classroom gardening (e.g. Earthbox gardening); harvesting lessons; cooking lessons and experimental kitchen activities utilising harvests; taste tests; nutrition-related education on food cultivation, healthy living skills, agriculture and nutrition science; physical education; healthy F\&V snack program; poster, poem and nutrition and vegetable charts displays on school boards, meat-free Monday, using locally source produce in school meals and market days to sell produce from the garden and local farmers' market visit. Outcomes of each study varied, but the majority primarily focused on the changes in children KAP on food consumption (particularly F\&V).

Description of the included studies 
A total of 35 studies have been included, and the characteristics of each intervention study are reported in Table 1 . There were 18 out of the 35 studies in which the intervention included parental involvement. In this review, the levels of parental involvement and associated activities differed between studies, ranging from students gardening with parents; student and family cooking events; parent gardening, home gardening, maintenance of school garden, school visit invitation to receive a brief of school gardening project, end-of-programme celebration invitation, takehome materials (e.g. "Family Stories" booklet and recipe cards) and parent newsletter (considered as weak parental component or low activity intensity). The main findings on the impact of SGBP are reported in Table 2. However, due to no significant difference in the outcome measures between the intervention with and without parental involvement, findings were combined and analysed all at once. 
Table 1

Summary of the included SGBP interventions $(n=35)$

\begin{tabular}{|c|c|c|c|c|c|c|c|}
\hline $\begin{array}{l}\text { Author (Year) } \\
\text { and location }\end{array}$ & $\begin{array}{l}\text { Duration/ } \\
\text { Study design }\end{array}$ & $\begin{array}{l}\text { Sample } \\
\text { size }\end{array}$ & $\begin{array}{l}\text { Sample } \\
\text { characteristics }\end{array}$ & $\begin{array}{l}\text { Parental } \\
\text { involvement }\end{array}$ & $\begin{array}{l}\text { Intervention } \\
\text { group }\end{array}$ & $\begin{array}{l}\text { Comparison or } \\
\text { control group }\end{array}$ & $\begin{array}{l}\text { Outcomes: } \\
\text { measurement } \\
\text { tools }\end{array}$ \\
\hline $\begin{array}{l}\text { Davis et al., } \\
\text { (2021) USA [1] }\end{array}$ & $\begin{array}{l}1 \text { year } \\
\text { cluster RCT }\end{array}$ & $\begin{array}{l}16 \\
\text { schools } \\
(n= \\
3135)\end{array}$ & $\begin{array}{l}8-11 \text { years } \\
\text { old }\end{array}$ & $\begin{array}{l}\text { Low }^{2} \\
9 \text { monthly parent } \\
\text { lessons: } 1 \text { hour } \\
\text { gardening, } \\
\text { nutrition and } \\
\text { cooking lessons } \\
\text { - The parent } \\
\text { curriculum also } \\
\text { included the } \\
\text { following topics; } \\
\text { importance of } \\
\text { family eating, } \\
\text { healthy } \\
\text { shopping, and } \\
\text { increasing home } \\
\text { available and } \\
\text { access of } \\
\text { healthy foods. }\end{array}$ & $\begin{array}{l}\text { Garden } \\
\text { Leadership } \\
\text { Committee } \\
\text { formation, } \\
\text { student } \\
\text { gardening, } \\
\text { nutrition, and } \\
\text { cooking lessons } \\
\text { (either a garden } \\
\text { taste-test ( } 7 \\
\text { lessons) or a } \\
\text { cooking activity), } \\
9 \text { monthly parent } \\
\text { lessons } \\
\text { ( } n=1412 \text { ) }\end{array}$ & $\begin{array}{l}\text { Compare: } \\
\text { delayed } \\
\text { intervention } \\
(n=1723)\end{array}$ & $\begin{array}{l}\text { 1) F\&V and } \\
\text { sugar } \\
\text { sweetened } \\
\text { beverage (SSB) } \\
\text { consumption: } \\
2015 \text { School } \\
\text { Physical Activity } \\
\text { and Nutrition } \\
\text { dietary screener } \\
\text { 2) Food and } \\
\text { meal choice } \\
\text { behaviours, self- } \\
\text { efficacy to cook } \\
\text { and/or prepare } \\
\text { F\&V and } \\
\text { gardening, } \\
\text { willingness to } \\
\text { try and } \\
\text { preferences for } \\
\text { F\&V, cooking } \\
\text { and gardening } \\
\text { attitudes, } \\
\text { nutrition and } \\
\text { gardening } \\
\text { knowledge, and } \\
\text { child food } \\
\text { security: } \\
\text { questionnaire }\end{array}$ \\
\hline $\begin{array}{l}\text { Barnard et al., } \\
\text { (2020) USA [2] }\end{array}$ & $\begin{array}{l}4 \text { years } \\
\text { pre-post } \\
\text { study }\end{array}$ & $\begin{array}{l}4 \\
\text { schools } \\
(n= \\
4300)\end{array}$ & $\begin{array}{l}2-19 \text { years } \\
\text { old }\end{array}$ & $\begin{array}{l}\text { High }^{1} \\
\text { Student and } \\
\text { family cooking } \\
\text { events }\end{array}$ & $\begin{array}{l}\text { School Gardens } \\
\text { and Classroom } \\
\text { Lessons ( } \mathrm{n}=172 \\
\text { lessons), Student } \\
\text { and Family } \\
\text { Cooking Events, } \\
\text { Carrot Camp ( }= \\
\text { 206), Sprout } \\
\text { Scouts }(\mathrm{n}=52)\end{array}$ & $\begin{array}{l}\text { No control } \\
\text { group } \\
(n=0)\end{array}$ & $\begin{array}{l}\text { 1) Knowledge, } \\
\text { attitudes, and } \\
\text { awareness of } \\
\text { the programme: } \\
\text { teacher survey } \\
\text { 2) } \\
\text { Parent/caregiver } \\
\text { knowledge and } \\
\text { attitudes related } \\
\text { to the } \\
\text { programme and } \\
\text { its potential } \\
\text { impact on } \\
\text { children: } \\
\text { parent/caregiver } \\
\text { survey } \\
\text { 3) F\&V } \\
\text { consumption: } \\
\text { parent/caregiver } \\
\text { survey }\end{array}$ \\
\hline
\end{tabular}




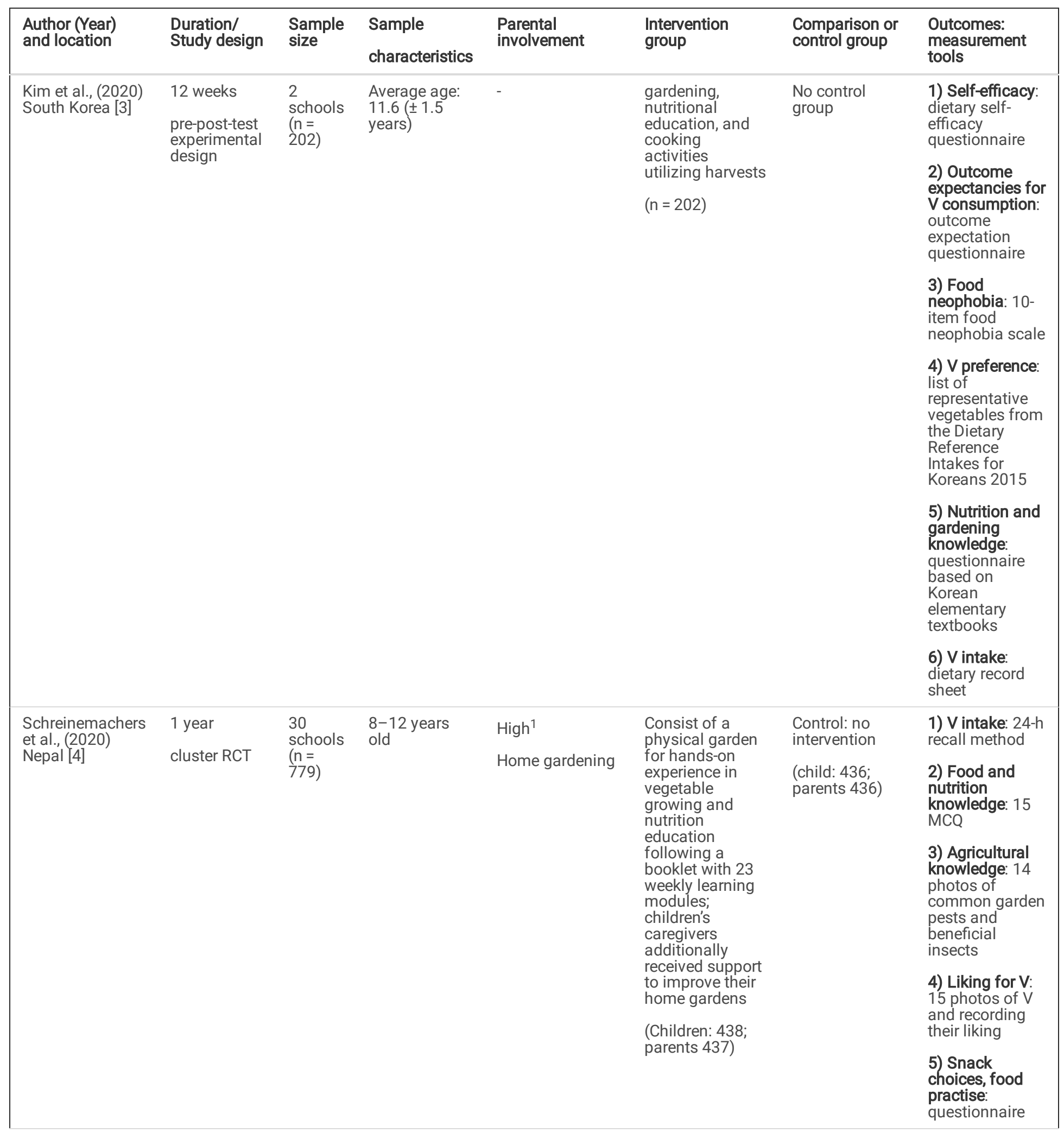




\begin{tabular}{|c|c|c|c|c|c|c|c|}
\hline $\begin{array}{l}\text { Author (Year) } \\
\text { and location }\end{array}$ & $\begin{array}{l}\text { Duration/ } \\
\text { Study design }\end{array}$ & $\begin{array}{l}\text { Sample } \\
\text { size }\end{array}$ & $\begin{array}{l}\text { Sample } \\
\text { characteristics }\end{array}$ & $\begin{array}{l}\text { Parental } \\
\text { involvement }\end{array}$ & $\begin{array}{l}\text { Intervention } \\
\text { group }\end{array}$ & $\begin{array}{l}\text { Comparison or } \\
\text { control group }\end{array}$ & $\begin{array}{l}\text { Outcomes: } \\
\text { measurement } \\
\text { tools }\end{array}$ \\
\hline $\begin{array}{l}\text { Shrestha et al., } \\
\text { (2020) Nepal [5] }\end{array}$ & $\begin{array}{l}5 \text { months } \\
\text { Cluster RCT }\end{array}$ & $\begin{array}{l}12 \\
\text { schools } \\
(n= \\
682)\end{array}$ & $\begin{array}{l}8-17 \text { years } \\
\text { old }\end{array}$ & - & $\begin{array}{l}\text { School } \\
\text { gardening } \\
\text { program } \\
(n=172)\end{array}$ & $\begin{array}{l}\text { Compare: } \\
\text { school garden } \\
\text { programme } \\
\text { with } \\
\text { complementary } \\
\text { WASH, health } \\
\text { and nutrition } \\
\text { interventions } \\
\text { ( } n=197) \\
\text { Control: no } \\
\text { intervention } \\
\text { ( } n=313 \text { ) }\end{array}$ & $\begin{array}{l}\text { 1) Dietary } \\
\text { intake: FFQ and } \\
\text { 24-h recall } \\
\text { questionnaire } \\
\text { 2) Nutrition } \\
\text { knowledge: } \\
\text { questionnaire }\end{array}$ \\
\hline $\begin{array}{l}\text { van den Berg et } \\
\text { al., (2020) USA } \\
\text { [6] }\end{array}$ & $\begin{array}{l}6 \text { months } \\
\text { non-RCT }\end{array}$ & $\begin{array}{l}28 \text { low- } \\
\text { income } \\
\text { schools } \\
(n= \\
1326)\end{array}$ & $\begin{array}{l}8-9 \text { years old } \\
\text { (42\% } \\
\text { Hispanic; } 78 \% \\
\text { free/reduced } \\
\text { lunch). }\end{array}$ & $\begin{array}{l}\text { Low }^{2} \\
\text { LEGE: gardens } \\
\text { built with } \\
\text { parents, took } \\
\text { home recipe card } \\
\text { and family } \\
\text { stories. } \\
\text { WAT! Program: } \\
\text { family } \\
\text { engagement } \\
\text { pieces (bonus } \\
\text { miles form), end- } \\
\text { of-program } \\
\text { celebration, } \\
\text { weekly English } \\
\text { and Spanish } \\
\text { newsletters } \\
\text { featuring both } \\
\text { healthy physical } \\
\text { and eating tips. }\end{array}$ & $\begin{array}{l}\text { (Learn! Grow! } \\
\text { Eat! Go! [LGEG]) } \\
\text { - school garden } \\
\text { \& school } \\
\text { curriculum } \\
(n=347)\end{array}$ & $\begin{array}{l}\text { Compare 1: } \\
\text { Physical } \\
\text { activity (PA) } \\
\text { intervention } \\
\text { (Walk Across } \\
\text { Texas [WAT!] } \\
\text { (n=336) } \\
\text { Compare 2: } \\
\text { both garden } \\
\text { and PA } \\
\text { intervention } \\
\text { (combined) (n } \\
=358 \text { ) } \\
\text { Control: } \\
\text { delayed } \\
\text { intervention (n } \\
=285 \text { ) }\end{array}$ & $\begin{array}{l}\text { 1) V preference, } \\
\text { nutrition } \\
\text { knowledge, } \\
\text { gardening with } \\
\text { parents, } \\
\text { previous day V } \\
\text { consumption: } \\
\text { student surveys } \\
\text { 2) Home V } \\
\text { availability: } \\
\text { parent surveys }\end{array}$ \\
\hline $\begin{array}{l}\text { Khan et al., } \\
\text { (2019) UK [7] }\end{array}$ & $\begin{array}{l}1 \text { school } \\
\text { year } \\
\text { mix method } \\
\text { study - } \\
\text { randomised } \\
\text { controlled }\end{array}$ & $\begin{array}{l}1 \\
\text { school } \\
(n=60)\end{array}$ & $\begin{array}{l}9-10 \text { years } \\
\text { old }\end{array}$ & - & $\begin{array}{l}\text { Gardening } \\
\text { intervention \& a } \\
\text { Meat-Free } \\
\text { Monday session, } \\
\text { PA \& knowledge } \\
\text { of nutrition } \\
(n=30)\end{array}$ & $\begin{array}{l}\text { Compare: } \\
\text { delayed } \\
\text { intervention } \\
(n=30)\end{array}$ & $\begin{array}{l}\text { 1) Attitude } \\
\text { towards, } \\
\text { frequency of } \\
\text { and preference } \\
\text { for eating F\&V: } \\
\text { self-report } \\
\text { questionnaire } \\
\text { 2) Experiences } \\
\text { of gardening } \\
\text { outdoors, } \\
\text { attitudes to } \\
\text { eating F\&V: } \\
\text { focus group } \\
\text { interview }\end{array}$ \\
\hline
\end{tabular}




\begin{tabular}{|c|c|c|c|c|c|c|c|}
\hline $\begin{array}{l}\text { Author (Year) } \\
\text { and location }\end{array}$ & $\begin{array}{l}\text { Duration/ } \\
\text { Study design }\end{array}$ & $\begin{array}{l}\text { Sample } \\
\text { size }\end{array}$ & $\begin{array}{l}\text { Sample } \\
\text { characteristics }\end{array}$ & $\begin{array}{l}\text { Parental } \\
\text { involvement }\end{array}$ & $\begin{array}{l}\text { Intervention } \\
\text { group }\end{array}$ & $\begin{array}{l}\text { Comparison or } \\
\text { control group }\end{array}$ & $\begin{array}{l}\text { Outcomes: } \\
\text { measurement } \\
\text { tools }\end{array}$ \\
\hline $\begin{array}{l}\text { Landry et al., } \\
\text { (2019) USA [8] }\end{array}$ & $\begin{array}{l}12 \text { weeks } \\
\text { RCT }\end{array}$ & $\begin{array}{l}4 \\
\text { schools } \\
(n= \\
290)\end{array}$ & $\begin{array}{l}\text { Low income, } \\
\text { primarily } \\
\text { Hispanic/ } \\
\text { Latino, } 8-11 \\
\text { years old }\end{array}$ & - & $\begin{array}{l}\text { LA Sprouts: } \\
\text { cooking and } \\
\text { nutrition } \\
\text { curriculum \& } \\
\text { gardening } \\
\text { curriculum } \\
(n=160)\end{array}$ & $\begin{array}{l}\text { Compare: } \\
\text { delayed } \\
\text { intervention } \\
(n=130)\end{array}$ & $\begin{array}{l}\text { 1) Dietary } \\
\text { intake: } 2007 \\
\text { Block Kids Food } \\
\text { Screeners } \\
\text { (adapted from } \\
\text { the Block Kids } \\
2004 \text { FFQ) } \\
\text { 2) Self-efficacy } \\
\text { to cook F\&V: } \\
\text { adapted } \\
\text { questionnaire } \\
\text { from } \\
\text { Baranowski et } \\
\text { al. } \\
\text { 3) Motivation to } \\
\text { cook and } \\
\text { garden F\&V: } \\
\text { motivation for } \\
\text { Health Behaving } \\
\text { from the } \\
\text { Treatment and } \\
\text { Self-Regulation } \\
\text { Questionnaire }\end{array}$ \\
\hline $\begin{array}{l}\text { Massarani et al., } \\
\text { (2019) Rio de } \\
\text { Janeiro [9] }\end{array}$ & $\begin{array}{l}3 \text { years } \\
\text { pre-post } \\
\text { study }\end{array}$ & $\begin{array}{l}1 \\
\text { school } \\
(n=89)\end{array}$ & $\begin{array}{l}11-12 \text { years } \\
\text { old adolescent } \\
\text { athletes (14- } \\
15 \text { years old } \\
\text { at follow up) }\end{array}$ & $\begin{array}{l}\text { Low }^{2} \\
\text { Building of } \\
\text { school garden } \\
\text { and experimental } \\
\text { kitchen with the } \\
\text { direct } \\
\text { involvement of } \\
\text { parents; } \\
\text { maintenance of } \\
\text { the garden } \\
\text { (organisation } \\
\text { and planning of } \\
\text { the school } \\
\text { garden); attend } \\
\text { the semi-annual } \\
\text { meeting }\end{array}$ & $\begin{array}{l}\text { School } \\
\text { gardening \& } \\
\text { experimental } \\
\text { kitchen activities } \\
\text { \& health } \\
\text { promotion class } \\
(\mathrm{n}=89)\end{array}$ & $\begin{array}{l}\text { No control } \\
\text { group }\end{array}$ & $\begin{array}{l}\text { 1) Dietary } \\
\text { intake: FFQ of } \\
12 \text { food items }\end{array}$ \\
\hline $\begin{array}{l}\text { Nele Huys et al., } \\
\text { (2019) Ghent } \\
{[10]}\end{array}$ & $\begin{array}{l}9 \text { weeks } \\
\text { non- } \\
\text { randomised } \\
\text { controlled }\end{array}$ & $\begin{array}{l}17 \\
\text { schools } \\
(n= \\
551)\end{array}$ & $\begin{array}{l}10-12 \text { years } \\
\text { old }\end{array}$ & - & $\begin{array}{l}\text { Gardening } \\
\text { activity: sowing, } \\
\text { taking care of } \\
\text { and harvesting } \\
\text { vegetables; } \\
\text { nutrition } \\
\text { education in } \\
\text { classroom } \\
(n=312)\end{array}$ & $\begin{array}{l}\text { Control: no } \\
\text { intervention } \\
(n=239)\end{array}$ & $\begin{array}{l}\text { 1) Children's V } \\
\text { intake and } \\
\text { determinants } \\
\text { (knowledge and } \\
\text { awareness): } \\
\text { questionnaires } \\
\text { 2) Program } \\
\text { evaluation: } \\
\text { process } \\
\text { evaluation } \\
\text { questionnaire } \\
\text { (teacher and } \\
\text { children) }\end{array}$ \\
\hline
\end{tabular}




\begin{tabular}{|c|c|c|c|c|c|c|c|}
\hline $\begin{array}{l}\text { Author (Year) } \\
\text { and location }\end{array}$ & $\begin{array}{l}\text { Duration/ } \\
\text { Study design }\end{array}$ & $\begin{array}{l}\text { Sample } \\
\text { size }\end{array}$ & $\begin{array}{l}\text { Sample } \\
\text { characteristics }\end{array}$ & $\begin{array}{l}\text { Parental } \\
\text { involvement }\end{array}$ & $\begin{array}{l}\text { Intervention } \\
\text { group }\end{array}$ & $\begin{array}{l}\text { Comparison or } \\
\text { control group }\end{array}$ & $\begin{array}{l}\text { Outcomes: } \\
\text { measurement } \\
\text { tools }\end{array}$ \\
\hline $\begin{array}{l}\text { Schreinemachers } \\
\text { et al., (2019) } \\
\text { Burkina Faso } \\
\text { [11] }\end{array}$ & $\begin{array}{l}1 \text { year } \\
\text { cluster RCT }\end{array}$ & $\begin{array}{l}30 \\
\text { schools } \\
(n= \\
1,760)\end{array}$ & $\begin{array}{l}8-14 \text { years } \\
\text { old }\end{array}$ & $\begin{array}{l}\text { High }^{1} \\
\text { Decided together } \\
\text { what vegetables } \\
\text { to grow in the } \\
\text { school garden; } \\
\text { helped to prepare } \\
\text { the school } \\
\text { garden and to } \\
\text { fence it with } \\
\text { locally available } \\
\text { materials; helped } \\
\text { to find water in } \\
\text { the dry season } \\
\text { and helped with } \\
\text { land preparation } \\
\text { and fencing. }\end{array}$ & $\begin{array}{l}\text { School } \\
\text { gardening; } \\
\text { complementary } \\
\text { agriculture, } \\
\text { nutrition and } \\
\text { WASH education; } \\
\text { local farmers } \\
\text { and other } \\
\text { community } \\
\text { members in } \\
\text { school garden } \\
\text { (2014: } n=500 ; \\
\text { 2015: } n=400)\end{array}$ & $\begin{array}{l}\text { Control: no } \\
\text { intervention } \\
(2014: \mathrm{n}=500 \text {; } \\
2015 \mathrm{n}=400)\end{array}$ & $\begin{array}{l}\text { 1) F\&V } \\
\text { preferences and } \\
\text { liking: rating for } \\
12 \mathrm{~V} \& 10 \text { snack } \\
\text { choices } \\
\text { 2) F\&V intake: } \\
\text { 24-hour recall } \\
\text { 3) F\&V } \\
\text { awareness: give } \\
\text { the correct } \\
\text { names of } 20 \\
\text { common F\&V } \\
\text { from colour } \\
\text { photos } \\
\text { 4) Food, } \\
\text { nutrition and } \\
\text { WASH } \\
\text { knowledge: test } \\
\text { adjusted from } \\
\text { Parmer et al. \& } \\
\text { Oldewage- } \\
\text { Theron and Egal } \\
\text { 5) Agriculture } \\
\text { knowledge: } \\
\text { photo test }\end{array}$ \\
\hline $\begin{array}{l}\text { Leuven et al., } \\
\text { (2018) } \\
\text { Netherlands [12] }\end{array}$ & $\begin{array}{l}7 \text { months } \\
\text { non- } \\
\text { randomised } \\
\text { controlled }\end{array}$ & $\begin{array}{l}3 \\
\text { schools } \\
(n= \\
150)\end{array}$ & $\begin{array}{l}10-12 \text { years } \\
\text { old }\end{array}$ & - & $\begin{array}{l}\text { Garden and } \\
\text { nutrition based } \\
\text { classroom } \\
\text { lessons, } 15 \\
\text { outdoor } \\
\text { gardening } \\
\text { lessons, and } 1 \\
\text { harvesting and } \\
\text { cooking lesson } \\
\text { Short term ( } \mathrm{n}= \\
\text { 106) } \\
\text { Long term ( } \mathrm{n}= \\
52)\end{array}$ & $\begin{array}{l}\text { Control: no } \\
\text { intervention } \\
(\mathrm{n}=65)\end{array}$ & $\begin{array}{l}\text { 1) Capability to } \\
\text { identify } V \text {, } \\
\text { preference for } \\
\text { those } V \text {, and the } \\
\text { opinion } \\
\text { regarding } V \text {, } \\
\text { gardening, and } \\
\text { outdoor activity: } \\
\text { questionnaire }\end{array}$ \\
\hline $\begin{array}{l}\text { Taylor et al., } \\
\text { (2018) USA [13] }\end{array}$ & $\begin{array}{l}1 \text { year } \\
\text { RCT }\end{array}$ & $\begin{array}{l}2 \\
\text { schools } \\
(n= \\
294)\end{array}$ & $\begin{array}{l}9-10 \text { years } \\
\text { old }\end{array}$ & $\begin{array}{l}\text { Low }^{2} \\
\text { Parent } \\
\text { newsletters }\end{array}$ & $\begin{array}{l}\text { Inquiry-based, } \\
\text { garden-enhanced } \\
\text { nutrition } \\
\text { curriculum, in- } \\
\text { class cooking } \\
\text { demonstrations, } \\
\text { take-home } \\
\text { activities, family } \\
\text { newsletters, a } \\
\text { health fair, and } \\
\text { school site- } \\
\text { specific wellness } \\
\text { committees } \\
\text { (n=161) }\end{array}$ & $\begin{array}{l}\text { Compare: } \\
\text { delayed } \\
\text { intervention } \\
(n=133)\end{array}$ & $\begin{array}{l}\text { 1) Dietary } \\
\text { intake: digital } \\
\text { images of } \\
\text { students' lunch } \\
\text { trays }\end{array}$ \\
\hline $\begin{array}{l}\text { Wells et al., } \\
\text { (2018) USA [14] }\end{array}$ & $\begin{array}{l}\text { 2-years } \\
\text { group RCT }\end{array}$ & $\begin{array}{l}46 \\
\text { schools } \\
(n= \\
2768)\end{array}$ & $\begin{array}{l}7-11 \text { years } \\
\text { old low- } \\
\text { income } \\
\text { children (8-12 } \\
\text { years old in } \\
\text { second year } \\
\text { intervention) }\end{array}$ & - & $\begin{array}{l}\text { School } \\
\text { gardening } \\
\text { activities and } \\
\text { nutrition } \\
\text { education } \\
(n=1491)\end{array}$ & $\begin{array}{l}\text { Control: no } \\
\text { intervention } \\
(n=1277)\end{array}$ & $\begin{array}{l}\text { 1) F\&V } \\
\text { availability at } \\
\text { home: modified } \\
\text { version of } \\
\text { Baranowski's } \\
\text { GEM-FJV } \\
\text { Availability } \\
\text { assessment }\end{array}$ \\
\hline $\begin{array}{l}\text { Gatto et al., } \\
\text { (2017) USA [15] }\end{array}$ & $\begin{array}{l}12 \text { weeks } \\
\text { RCT }\end{array}$ & $\begin{array}{l}4 \\
\text { schools } \\
(n= \\
375)\end{array}$ & $\begin{array}{l}\text { Low income, } \\
\text { primarily } \\
\text { Hispanic/ } \\
\text { Latino, 8-11 } \\
\text { years old }\end{array}$ & $\begin{array}{l}\text { Low }^{2} \\
\text { Bimonthly } \\
\text { cooking/nutrition } \\
\text { and gardening } \\
\text { classes offered } \\
\text { to the parents }\end{array}$ & $\begin{array}{l}\text { LA Sprouts: } 45- \\
\text { min interactive } \\
\text { cooking/nutrition } \\
\text { lesson \& 45-min } \\
\text { gardening lesson } \\
(n=172)\end{array}$ & $\begin{array}{l}\text { Compare: } \\
\text { delayed } \\
\text { intervention } \\
(n=147)\end{array}$ & $\begin{array}{l}\text { 1) Dietary } \\
\text { intake: } 2007 \\
\text { Block Kids Food } \\
\text { Screeners } \\
\text { (adapted from } \\
\text { the Block Kids } \\
2004 \text { FFQ) }\end{array}$ \\
\hline
\end{tabular}




\begin{tabular}{|c|c|c|c|c|c|c|c|}
\hline $\begin{array}{l}\text { Author (Year) } \\
\text { and location }\end{array}$ & $\begin{array}{l}\text { Duration/ } \\
\text { Study design }\end{array}$ & $\begin{array}{l}\text { Sample } \\
\text { size }\end{array}$ & $\begin{array}{l}\text { Sample } \\
\text { characteristics }\end{array}$ & $\begin{array}{l}\text { Parental } \\
\text { involvement }\end{array}$ & $\begin{array}{l}\text { Intervention } \\
\text { group }\end{array}$ & $\begin{array}{l}\text { Comparison or } \\
\text { control group }\end{array}$ & $\begin{array}{l}\text { Outcomes: } \\
\text { measurement } \\
\text { tools }\end{array}$ \\
\hline $\begin{array}{l}\text { Lee et al., (2017) } \\
\text { USA [16] }\end{array}$ & $\begin{array}{l}6 \text { weeks (12 } \\
\text { one-hour } \\
\text { sessions) } \\
\text { pre-post } \\
\text { study }\end{array}$ & $\begin{array}{l}6 \\
\text { centres } \\
(n=89)\end{array}$ & $3-5$ years old & $\begin{array}{l}\text { Low }^{2} \\
3 \text { newsletters } \\
\text { (health benefits } \\
\text { of adequate F\&V } \\
\text { intake, strategies } \\
\text { for improving } \\
\text { home intake); } \\
\text { encouraged to } \\
\text { complete } \\
\text { activities with } \\
\text { children - } \\
\text { selecting a } \\
\text { favourite recipe } \\
\text { for a class recipe } \\
\text { book }\end{array}$ & $\begin{array}{l}\text { Lessons include } \\
\text { songs, games, } \\
\text { and interactive } \\
\text { learning } \\
\text { activities } \\
\text { involving garden } \\
\text { maintenance } \\
\text { and taste tests } \\
(n=89)\end{array}$ & $\begin{array}{l}\text { No control } \\
\text { group }\end{array}$ & $\begin{array}{l}\text { 1) Dietary } \\
\text { intake: non- } \\
\text { consecutive 3- } \\
\text { day food } \\
\text { records from } \\
\text { parents } \\
\text { 2) F\&V } \\
\text { Availability } \\
\text { (Home): the F\&V } \\
\text { Home } \\
\text { Availability } \\
\text { questionnaire }\end{array}$ \\
\hline $\begin{array}{l}\text { Schreinemachers } \\
\text { et al., (2017) } \\
\text { Bhutan [17] }\end{array}$ & $\begin{array}{l}1 \text { year } \\
\text { cluster RCT }\end{array}$ & $\begin{array}{l}18 \\
\text { schools } \\
(n= \\
517)\end{array}$ & $\begin{array}{l}9-15 \text { years } \\
\text { old }\end{array}$ & $\begin{array}{l}\text { High }{ }^{1} \\
\text { Cultivation of } \\
\text { vegetables with } \\
\text { children; } \\
\text { provided advice, } \\
\text { gardening tools } \\
\text { and other } \\
\text { materials and } \\
\text { advised school } \\
\text { teachers on } \\
\text { crops and } \\
\text { varieties to grow. } \\
\text { Teachers visited } \\
\text { the parents at } \\
\text { home and } \\
\text { encouraged } \\
\text { home gardening }\end{array}$ & $\begin{array}{l}\text { School garden; } \\
\text { weekly lessons } \\
\text { in gardening, } \\
\text { nutrition, and } \\
\text { water, sanitation } \\
\text { and hygiene } \\
\text { (WASH); } \\
\text { promotion } \\
\text { activities: poster } \\
\text { displays, poem } \\
\text { displays on } \\
\text { school boards, } \\
\text { songs, nutrition } \\
\text { charts, vegetable } \\
\text { charts, pledges } \\
\text { (2014: no data; } \\
\text { 2015: } \mathrm{n}=260 \text { ) }\end{array}$ & $\begin{array}{l}\text { Control: no } \\
\text { intervention } \\
\text { (2014: no data; } \\
\text { 2015: } \mathrm{n}=265)\end{array}$ & $\begin{array}{l}\text { 1) F\&V } \\
\text { preferences and } \\
\text { liking: rating for } \\
12 \mathrm{~V}+10 \text { snack } \\
\text { choices } \\
\text { 2) F\&V intake: } \\
\text { 24-hour recall } \\
\text { 3) F\&V } \\
\text { awareness: give } \\
\text { the correct } \\
\text { names of } 20 \\
\text { common F\&V } \\
\text { from colour } \\
\text { photos } \\
\text { 4) Food, }\end{array}$ \\
\hline $\begin{array}{l}\text { Schreinemachers } \\
\text { et al., (2017) } \\
\text { Nepal [18] }\end{array}$ & $\begin{array}{l}1 \text { year } \\
\text { cluster RCT }\end{array}$ & $\begin{array}{l}30 \\
\text { schools } \\
(n= \\
1370)\end{array}$ & $\begin{array}{l}10-15 \text { years } \\
\text { old, low } \\
\text { income }\end{array}$ & & $\begin{array}{l}\text { A school garden, } \\
\text { gardening, } \\
\text { nutrition and } \\
\text { WASH education } \\
\text { and promotional } \\
\text { materials for } \\
\text { children and } \\
\text { parents (poster } \\
\text { display, } \\
\text { distribution of } \\
\text { handouts about } \\
\text { nutritious food } \\
\text { and hand } \\
\text { washing) } \\
\text { (2014: } n=429 ; \\
2015: n=369)\end{array}$ & $\begin{array}{l}\text { Control: no } \\
\text { intervention } \\
(2014: \mathrm{n}=846 \\
\text { 2015: } \mathrm{n}=416)\end{array}$ & $\begin{array}{l}\text { WASH } \\
\text { knowledge: test } \\
\text { adjusted from } \\
\text { Parmer et al. \& } \\
\text { Oldewage- } \\
\text { Theron and Egal } \\
\text { 5) Agriculture } \\
\text { knowledge: } \\
\text { photo test }\end{array}$ \\
\hline $\begin{array}{l}\text { Davis et al., } \\
\text { (2016) USA [19] }\end{array}$ & $\begin{array}{l}12 \text { weeks } \\
\text { RCT }\end{array}$ & $\begin{array}{l}4 \\
\text { schools } \\
(n= \\
304)\end{array}$ & $\begin{array}{l}\text { Low income, } \\
\text { primarily } \\
\text { Hispanic/ } \\
\text { Latino, 8-11 } \\
\text { years old }\end{array}$ & - & $\begin{array}{l}\text { LA Sprouts } \\
\text { participants: } \\
\text { weekly 45- } \\
\text { minute } \\
\text { interactive } \\
\text { cooking and } \\
\text { nutrition } \\
\text { education } \\
\text { lesson, 45- } \\
\text { minute } \\
\text { interactive } \\
\text { gardening } \\
\text { lesson, and visits } \\
\text { to a local } \\
\text { farmers' market } \\
4 \text { times during } \\
\text { intervention } \\
\text { (n=167) }\end{array}$ & $\begin{array}{l}\text { Compare: } \\
\text { delayed } \\
\text { intervention } \\
(n=137)\end{array}$ & $\begin{array}{l}\text { 1) F\&V } \\
\text { preferences and } \\
\text { identification; } \\
\text { self-efficacy to } \\
\text { eat, cook and } \\
\text { garden F\&V; } \\
\text { nutrition \& } \\
\text { gardening } \\
\text { knowledge, } \\
\text { attitudes about } \\
\text { cooking and } \\
\text { gardening \& } \\
\text { current home } \\
\text { gardening } \\
\text { practices; } \\
\text { willingness to } \\
\text { try F\&V: } \\
\text { questionnaire } \\
\text { 2) Motivation to } \\
\text { eat, cook F\&V \& } \\
\text { gardening: } \\
\text { Treatment and } \\
\text { Self-Regulation } \\
\text { Questionnaire }\end{array}$ \\
\hline
\end{tabular}




\begin{tabular}{|c|c|c|c|c|c|c|c|}
\hline $\begin{array}{l}\text { Author (Year) } \\
\text { and location }\end{array}$ & $\begin{array}{l}\text { Duration/ } \\
\text { Study design }\end{array}$ & $\begin{array}{l}\text { Sample } \\
\text { size }\end{array}$ & $\begin{array}{l}\text { Sample } \\
\text { characteristics }\end{array}$ & $\begin{array}{l}\text { Parental } \\
\text { involvement }\end{array}$ & $\begin{array}{l}\text { Intervention } \\
\text { group }\end{array}$ & $\begin{array}{l}\text { Comparison or } \\
\text { control group }\end{array}$ & $\begin{array}{l}\text { Outcomes: } \\
\text { measurement } \\
\text { tools }\end{array}$ \\
\hline $\begin{array}{l}\text { Duncan et al., } \\
\text { (2015) UK [20] }\end{array}$ & $\begin{array}{l}12 \text { week } \\
\text { non- } \\
\text { randomised } \\
\text { controlled }\end{array}$ & $\begin{array}{l}2 \\
\text { schools } \\
(n=77)\end{array}$ & $\begin{array}{l}4-11 \text { years } \\
\text { old }\end{array}$ & - & $\begin{array}{l}\text { Theory-based } \\
\text { intervention - a } \\
\text { school garden, } \\
\text { cooking lessons, } \\
\text { exploring plants } \\
\text { and growth in } \\
\text { science and } \\
\text { literacy } \\
(n=46)\end{array}$ & $\begin{array}{l}\text { Control: no } \\
\text { intervention } \\
(n=31)\end{array}$ & $\begin{array}{l}\text { 1) F\&V } \\
\text { consumption: } \\
\text { measures of the } \\
\text { TPB related to } \\
\text { F\&V } \\
\text { consumption } \\
\text { 2) F\&V } \\
\text { consumption } \\
\text { behaviour: Day } \\
\text { in the Life } \\
\text { Questionnaire } \\
\text { (DILQ) }\end{array}$ \\
\hline $\begin{array}{l}\text { Hanbazaza et al., } \\
\text { (2015) Canada } \\
\text { [21] }\end{array}$ & $\begin{array}{l}2 \text { school } \\
\text { years } \\
\text { pre-post } \\
\text { study }\end{array}$ & $\begin{array}{l}1 \\
\text { school } \\
(n= \\
116)\end{array}$ & $\begin{array}{l}6-12 \text { years } \\
\text { old }\end{array}$ & - & $\begin{array}{l}\text { Classroom } \\
\text { gardening (Earth } \\
\text { Box container } \\
\text { gardening) \& a } \\
\text { healthy F\&V } \\
\text { snack program } \\
(n=66)\end{array}$ & $\begin{array}{l}\text { No control } \\
\text { group }\end{array}$ & $\begin{array}{l}\text { 1) Children's } \\
\text { knowledge of } \\
\text { F\&V: asking } \\
\text { children to write } \\
\text { down } 5 \text { V\&F that } \\
\text { they knew } \\
\text { 2) F\&V } \\
\text { preferences and } \\
\text { home } \\
\text { consumption: } \\
\text { an adapted } \\
\text { questionnaire }\end{array}$ \\
\hline $\begin{array}{l}\text { Sharma et al., } \\
\text { (2015) USA [22] }\end{array}$ & $\begin{array}{l}8 \text { weeks } \\
\text { pre-post } \\
\text { study }\end{array}$ & $\begin{array}{l}2 \\
\text { centres } \\
(n= \\
103)\end{array}$ & $3-5$ years old & $\begin{array}{l}\text { Low }^{2} \\
\text { Children took } \\
\text { their plants } \\
\text { home to share } \\
\text { with their } \\
\text { families to } \\
\text { encourage } \\
\text { dialogue with } \\
\text { parents; invite } \\
\text { parent to End-of- } \\
\text { program } \\
\text { celebration }\end{array}$ & $\begin{array}{l}8 \text { PLANT Garden } \\
\text { lessons (teacher- } \\
\text { led) with hands- } \\
\text { on activities } \\
\text { emphasizing } \\
\text { gardening and } \\
\text { nutrition } \\
(n=103)\end{array}$ & $\begin{array}{l}\text { No control } \\
\text { group }\end{array}$ & $\begin{array}{l}\text { 1) Preference } \\
\text { and willingness } \\
\text { to try F\&Vs } \\
\text { among pre- } \\
\text { schoolers: pre- } \\
\text { post self- } \\
\text { reported parent } \\
\text { surveys }\end{array}$ \\
\hline $\begin{array}{l}\text { Spears-Lanoix et } \\
\text { al.,(2015) USA } \\
\text { [23] }\end{array}$ & $\begin{array}{l}5 \text { months } \\
\text { pre-post } \\
\text { study }\end{array}$ & $\begin{array}{l}1 \\
\text { school } \\
(n=44)\end{array}$ & $8-9$ years old & $\begin{array}{l}\text { High }^{1} \\
\text { Gardening } \\
\text { together, snacks } \\
\text { and meals } \\
\text { together, dinners } \\
\text { eaten together, } \\
\text { and doing } \\
\text { physical activity } \\
\text { together. }\end{array}$ & $\begin{array}{l}\text { JMG: youth } \\
\text { horticulture } \\
\text { classroom } \\
\text { curriculum - } \\
\text { building a class } \\
\text { garden, growing } \\
\text { seven V, tasting } \\
\text { and rating each } \\
\text { V, raw, and } \\
\text { participating in V } \\
\text { recipe tasting }\end{array}$ & $\begin{array}{l}\text { Compare: WAT } \\
\text { (PA } \\
\text { intervention) - } \\
\text { family bonus } \\
\text { miles, waling } \\
\text { Bingo, and } \\
\text { class activity } \\
\text { breaks } \\
\text { (Children: 44, } \\
\text { Parents: } 34 \text { ) }\end{array}$ & $\begin{array}{l}\text { 1) Food } \\
\text { availability: } \\
\text { parent survey } \\
\text { 2) V preference } \\
\text { and } \\
\text { consumption: } \\
\text { student self- } \\
\text { reported } \\
\text { questionnaire } \\
\text { 3) Nutrition and } \\
\text { health } \\
\text { knowledge: } \\
\text { questions about } \\
\text { healthy living }\end{array}$ \\
\hline $\begin{array}{l}\text { Wells et al., } \\
\text { (2015) USA [24] }\end{array}$ & $\begin{array}{l}\text { 2-years (40 } \\
\text { lessons) } \\
\text { RCT }\end{array}$ & $\begin{array}{l}49 \\
\text { schools } \\
(n= \\
3,061)\end{array}$ & $\begin{array}{l}6-12 \text { years } \\
\text { old }\end{array}$ & - & $\begin{array}{l}\text { Nutrition and } \\
\text { garden-based } \\
\text { lessons \& } \\
\text { gardening } \\
\text { activities } \\
(n=1622)\end{array}$ & $\begin{array}{l}\text { Control: no } \\
\text { intervention } \\
(n=1439)\end{array}$ & $\begin{array}{l}\text { 1) Science } \\
\text { knowledge: 7- } \\
\text { item MCQ } \\
\text { selected from } \\
\text { the University of } \\
\text { Missouri (UM) } \\
\text { 'Eating from the } \\
\text { Garden } \\
\text { Curriculum' } \\
\text { survey }\end{array}$ \\
\hline
\end{tabular}




\begin{tabular}{|c|c|c|c|c|c|c|c|}
\hline $\begin{array}{l}\text { Author (Year) } \\
\text { and location }\end{array}$ & $\begin{array}{l}\text { Duration/ } \\
\text { Study design }\end{array}$ & $\begin{array}{l}\text { Sample } \\
\text { size }\end{array}$ & $\begin{array}{l}\text { Sample } \\
\text { characteristics }\end{array}$ & $\begin{array}{l}\text { Parental } \\
\text { involvement }\end{array}$ & $\begin{array}{l}\text { Intervention } \\
\text { group }\end{array}$ & $\begin{array}{l}\text { Comparison or } \\
\text { control group }\end{array}$ & $\begin{array}{l}\text { Outcomes: } \\
\text { measurement } \\
\text { tools }\end{array}$ \\
\hline $\begin{array}{l}\text { Bontrager Yoder } \\
\text { et al., (2014) USA } \\
\text { [25] }\end{array}$ & $\begin{array}{l}1 \text { year } \\
\text { quasi- } \\
\text { experimental } \\
\text { baseline and } \\
\text { follow-up } \\
\text { assessments }\end{array}$ & $\begin{array}{l}9 \\
\text { schools } \\
(n= \\
1,117)\end{array}$ & $\begin{array}{l}8-11 \text { years } \\
\text { old }\end{array}$ & - & $\begin{array}{l}\text { Farm to School } \\
\text { programme: } \\
\text { Harvest of the } \\
\text { Month, school } \\
\text { garden, locally } \\
\text { sourced produce } \\
\text { in school meals } \\
\text { \& classroom } \\
\text { lessons } \\
(n=1117)\end{array}$ & $\begin{array}{l}\text { No control } \\
\text { group }\end{array}$ & $\begin{array}{l}\text { 1) Knowledge of } \\
\text { food, nutrition, } \\
\text { and agriculture; } \\
\text { attitudes toward } \\
\text { trying FV; } \\
\text { perception/self- } \\
\text { efficacy for } \\
\text { eating } \\
\text { healthfully, and } \\
\text { preference for, } \\
\text { exposure of and } \\
\text { willingness to } \\
\text { try F\&V: } \\
\text { questionnaire } \\
\text { 2) Dietary } \\
\text { intake: FFQ, } \\
\text { lunch tray photo } \\
\text { observation }\end{array}$ \\
\hline $\begin{array}{l}\text { Cotter et al., } \\
\text { (2013) Portugal } \\
{[26]}\end{array}$ & $\begin{array}{l}6 \text { months } \\
\text { cluster RCT }\end{array}$ & $\begin{array}{l}1 \\
\text { school } \\
(n= \\
155)\end{array}$ & $\begin{array}{l}10-12 \text { years } \\
\text { old }\end{array}$ & - & $\begin{array}{l}\text { Lessons } \\
\text { (dangers of high } \\
\text { salt intake), } \\
\text { gardening } \\
\text { activities and } \\
\text { collection of } \\
\text { herbs for salt } \\
\text { substitute at } \\
\text { home } \\
(n=58)\end{array}$ & $\begin{array}{l}\text { Compare: } \\
\text { weekly lessons } \\
\text { about the } \\
\text { dangers of } \\
\text { high salt intake } \\
(n=47) \\
\text { Control: no } \\
\text { intervention } \\
(n=34)\end{array}$ & $\begin{array}{l}\text { 1) Estimated } \\
\text { salt intake: } \\
\text { derived from } 24- \\
\text { h urinary } \\
\text { collection ( } 1 \\
\mathrm{mEq} / 24 \mathrm{~h} \\
\text { sodium } 1 / 4^{1 / 4} \\
0.058 \mathrm{~g} \text { per day } \\
\text { salt) }\end{array}$ \\
\hline $\begin{array}{l}\text { Gibbs et al., } \\
\text { (2013) Australia } \\
{[27]}\end{array}$ & $\begin{array}{l}2.5 \text { years } \\
\text { pre-post } \\
\text { study }\end{array}$ & $\begin{array}{l}12 \\
\text { schools } \\
(n= \\
764)\end{array}$ & $\begin{array}{l}8-12 \text { years } \\
\text { old }\end{array}$ & $\begin{array}{l}\text { Low }^{2} \\
\text { Did not specified }\end{array}$ & $\begin{array}{l}\text { Garden class } \\
\text { and kitchen } \\
\text { class } \\
(n=764)\end{array}$ & $\begin{array}{l}\text { No control } \\
\text { group }\end{array}$ & $\begin{array}{l}\text { 1) Willingness } \\
\text { to try new foods, } \\
\text { knowledge \& } \\
\text { capacity to } \\
\text { describe foods, } \\
\text { and healthy } \\
\text { eating: separate } \\
\text { focus group } \\
\text { discussions, } \\
\text { parent and child } \\
\text { questionnaire }\end{array}$ \\
\hline $\begin{array}{l}\text { Gatto et al., } \\
\text { (2012) USA [28] }\end{array}$ & $\begin{array}{l}12 \text { weeks } \\
\text { non-RCT }\end{array}$ & $\begin{array}{l}1 \\
\text { school } \\
(n= \\
104)\end{array}$ & $\begin{array}{l}\text { Latino } 9-11 \\
\text { years old, } \\
\text { more than } \\
\text { half were } \\
\text { overweight or } \\
\text { obese }\end{array}$ & $\begin{array}{l}\text { Low }^{2} \\
\text { Did not specified }\end{array}$ & $\begin{array}{l}\text { LA Sprouts } \\
\text { participants: } \\
\text { weekly 45- } \\
\text { minute } \\
\text { interactive } \\
\text { cooking and } \\
\text { nutrition } \\
\text { education } \\
\text { lesson, 45- } \\
\text { minute } \\
\text { interactive } \\
\text { gardening lesson } \\
\text { and visits to a } \\
\text { local farmers' } \\
\text { market } 4 \text { times } \\
\text { during } \\
\text { intervention } \\
\text { (n=34) }\end{array}$ & $\begin{array}{l}\text { Compare: } \\
\text { delayed } \\
\text { intervention } \\
(n=70)\end{array}$ & $\begin{array}{l}\text { 1) Motivation } \\
\text { for healthy } \\
\text { eating: } \\
\text { Treatment and } \\
\text { Self-Regulation } \\
\text { Questionnaire } \\
\text { 2) Attitudes } \\
\text { About, } \\
\text { Preferences for, } \\
\text { Perceptions, and } \\
\text { Self-Efficacy to } \\
\text { Eat and Cook } \\
\text { F\&V: validated } \\
\text { questionnaire }\end{array}$ \\
\hline
\end{tabular}




\begin{tabular}{|c|c|c|c|c|c|c|c|}
\hline $\begin{array}{l}\text { Author (Year) } \\
\text { and location }\end{array}$ & $\begin{array}{l}\text { Duration/ } \\
\text { Study design }\end{array}$ & $\begin{array}{l}\text { Sample } \\
\text { size }\end{array}$ & $\begin{array}{l}\text { Sample } \\
\text { characteristics }\end{array}$ & $\begin{array}{l}\text { Parental } \\
\text { involvement }\end{array}$ & $\begin{array}{l}\text { Intervention } \\
\text { group }\end{array}$ & $\begin{array}{l}\text { Comparison or } \\
\text { control group }\end{array}$ & $\begin{array}{l}\text { Outcomes: } \\
\text { measurement } \\
\text { tools }\end{array}$ \\
\hline $\begin{array}{l}\text { Jaenke et al., } \\
\text { (2012) Australia } \\
{[29]}\end{array}$ & $\begin{array}{l}10 \text { weeks } \\
\text { pre-post } \\
\text { study }\end{array}$ & $\begin{array}{l}2 \\
\text { schools } \\
(n= \\
127)\end{array}$ & $\begin{array}{l}11-12 \text { years } \\
\text { old }\end{array}$ & $\begin{array}{l}\text { Low }^{2} \\
3 \text { newsletters } \\
\text { (health benefits } \\
\text { of adequate F\&V } \\
\text { intake, strategies } \\
\text { for improving } \\
\text { home intake); } \\
\text { encouraged to } \\
\text { complete } \\
\text { activities with } \\
\text { their children - } \\
\text { selecting a } \\
\text { favourite recipe } \\
\text { for a class recipe } \\
\text { book }\end{array}$ & $\begin{array}{l}\text { Nutrition } \\
\text { education \& } \\
\text { gardening } \\
\text { intervention (with } \\
\text { kitchen-based } \\
\text { activities) } \\
(n=35)\end{array}$ & $\begin{array}{l}\text { Compare: } \\
\text { nutrition } \\
\text { education only } \\
(\mathrm{n}=35) \\
\text { Control: no } \\
\text { intervention ( } \mathrm{n} \\
=57)\end{array}$ & $\begin{array}{l}\text { 1) Food } \\
\text { preference, } \\
\text { willingness to } \\
\text { taste: } \\
\text { questionnaire + } \\
\text { five-item food } \\
\text { preference } \\
\text { assessment too } \\
\text { 2) F\&V intake: } 2 \\
\text { repeat 24-hour } \\
\text { recalls }\end{array}$ \\
\hline $\begin{array}{l}\text { Davis et al., } \\
\text { (2011) USA [30] }\end{array}$ & $\begin{array}{l}12 \text { weeks } \\
\text { non-RCT }\end{array}$ & $\begin{array}{l}1 \\
\text { school } \\
(n= \\
104)\end{array}$ & $\begin{array}{l}\text { Latino } 9-11 \\
\text { years old, } \\
\text { more than } \\
\text { half were } \\
\text { overweight or } \\
\text { obese }\end{array}$ & $\begin{array}{l}\text { Low }^{2} \\
\text { Three separate } \\
60 \text {-minute } \\
\text { parental nutrition } \\
\text { and gardening } \\
\text { classes }\end{array}$ & $\begin{array}{l}\text { LA Sprouts } \\
\text { participants: } \\
\text { weekly 45- } \\
\text { minute } \\
\text { interactive } \\
\text { cooking and } \\
\text { nutrition } \\
\text { education } \\
\text { lesson, 45- } \\
\text { minute } \\
\text { interactive } \\
\text { gardening lesson } \\
\text { and visits to a } \\
\text { local farmers' } \\
\text { market } 4 \text { times } \\
\text { during } \\
\text { intervention } \\
\text { ( } \mathrm{n}=34 \text { ) }\end{array}$ & $\begin{array}{l}\text { Compare: } \\
\text { delayed } \\
\text { intervention } \\
(n=70)\end{array}$ & $\begin{array}{l}\text { 1) Dietary } \\
\text { intake: } 2007 \\
\text { Block Kids Food } \\
\text { Screeners } \\
\text { (adapted from } \\
\text { the Block Kids } \\
2004 \text { FFQ) }\end{array}$ \\
\hline $\begin{array}{l}\text { Ratcliffe et al., } \\
\text { (2011) USA [31] }\end{array}$ & $\begin{array}{l}13 \text { weeks } \\
\text { non- } \\
\text { randomised } \\
\text { controlled }\end{array}$ & $\begin{array}{l}3 \\
\text { schools } \\
(n= \\
320)\end{array}$ & $\begin{array}{l}11-13 \text { years } \\
\text { old }\end{array}$ & - & $\begin{array}{l}\text { Hands-on } \\
\text { gardening \& } \\
\text { garden-based } \\
\text { learning } \\
\text { activities } \\
\text { integrated into } \\
\text { science lesson } \\
(n=170)\end{array}$ & $\begin{array}{l}\text { Compare: only } \\
\text { garden-based } \\
\text { sessions } \\
\text { integrated into } \\
\text { science class } \\
(n=150)\end{array}$ & $\begin{array}{l}\text { 1)Knowledge, } \\
\text { attitude and } \\
\text { behaviour: } \\
\text { Garden } \\
\text { Vegetables } \\
\text { Frequency } \\
\text { Questionnaire } \\
\text { (GVFQ) and the } \\
\text { Taste Test; } \\
\text { 2)Vegetable } \\
\text { consumption: } \\
\text { 24-hr recall }\end{array}$ \\
\hline $\begin{array}{l}\text { Morgan et al. } \\
(2010) \text { Australia } \\
{[32]}\end{array}$ & $\begin{array}{l}10 \text { weeks } \\
\text { non-RCT }\end{array}$ & $\begin{array}{l}2 \\
\text { schools } \\
(n= \\
127)\end{array}$ & $\begin{array}{l}11-12 \text { years } \\
\text { old }\end{array}$ & $\begin{array}{l}\text { Low }^{2} \\
3 \text { newsletters } \\
\text { (health benefits } \\
\text { of adequate F\&V } \\
\text { intake, strategies } \\
\text { for improving } \\
\text { home intake); } \\
\text { encouraged to } \\
\text { complete } \\
\text { activities with } \\
\text { their children - } \\
\text { selecting a } \\
\text { favourite recipe } \\
\text { for a class recipe } \\
\text { book }\end{array}$ & $\begin{array}{l}\text { Nutrition } \\
\text { education \& } \\
\text { gardening } \\
\text { intervention (with } \\
\text { kitchen-based } \\
\text { activities) } \\
(n=35)\end{array}$ & $\begin{array}{l}\text { Compare: } \\
\text { nutrition } \\
\text { education only } \\
(\mathrm{n}=35) \\
\text { Control: no } \\
\text { intervention ( } \mathrm{n} \\
=57)\end{array}$ & $\begin{array}{l}\text { 1) F\&V intake: } \\
24 \text { h recall } \\
\text { 2) V preference: } \\
\text { 'taste and rate' } \\
\text { methods } \\
\text { developed by } \\
\text { Birch and } \\
\text { Sullivan } \\
\text { 3) F\&V } \\
\text { knowledge: } \\
\text { questionnaire } \\
\text { used in US } \\
\text { 'Gimme 5' } \\
\text { intervention } \\
\text { 4) Quality of } \\
\text { school life: the } \\
\text { quality of } \\
\text { school life } \\
\text { (QoSL) } \\
\text { instrument " }\end{array}$ \\
\hline
\end{tabular}




\begin{tabular}{|c|c|c|c|c|c|c|c|}
\hline $\begin{array}{l}\text { Author (Year) } \\
\text { and location }\end{array}$ & $\begin{array}{l}\text { Duration/ } \\
\text { Study design }\end{array}$ & $\begin{array}{l}\text { Sample } \\
\text { size }\end{array}$ & $\begin{array}{l}\text { Sample } \\
\text { characteristics }\end{array}$ & $\begin{array}{l}\text { Parental } \\
\text { involvement }\end{array}$ & $\begin{array}{l}\text { Intervention } \\
\text { group }\end{array}$ & $\begin{array}{l}\text { Comparison or } \\
\text { control group }\end{array}$ & $\begin{array}{l}\text { Outcomes: } \\
\text { measurement } \\
\text { tools }\end{array}$ \\
\hline $\begin{array}{l}\text { Parmer et al., } \\
\text { (2009) USA [33] }\end{array}$ & $\begin{array}{l}28 \text { weeks } \\
\text { non- } \\
\text { randomised } \\
\text { controlled }\end{array}$ & $\begin{array}{l}1 \\
\text { school } \\
(n= \\
115)\end{array}$ & $7-8$ years old & - & $\begin{array}{l}\text { Nutrition } \\
\text { education \& } \\
\text { gardening } \\
\text { intervention } \\
(n=39)\end{array}$ & $\begin{array}{l}\text { Compare: } \\
\text { nutrition } \\
\text { education only } \\
(n=37) \\
\text { Control: no } \\
\text { intervention } \\
(n=39)\end{array}$ & $\begin{array}{l}\text { 1) F\&V intake: } \\
\text { F\&V survey; } \\
\text { lunchroom } \\
\text { observation } \\
\text { 2) F\&V } \\
\text { preference: F\&V } \\
\text { preference } \\
\text { questionnaire, } \\
\text { lunchroom } \\
\text { observation } \\
\text { 3) Nutrition } \\
\text { knowledge: } \\
\text { questionnaire }\end{array}$ \\
\hline $\begin{array}{l}\text { Somerset et al., } \\
\text { (2009) Australia } \\
{[34]}\end{array}$ & $\begin{array}{l}12 \text { months } \\
\text { intervention } \\
\text { trial }\end{array}$ & $\begin{array}{l}1 \\
\text { school } \\
(n= \\
252)\end{array}$ & $\begin{array}{l}\text { 9-13 years } \\
\text { old in a low } \\
\text { socio- } \\
\text { economic } \\
\text { area }\end{array}$ & - & $\begin{array}{l}\text { Introduction of a } \\
\text { school-based } \\
\text { food garden, }(n= \\
130)\end{array}$ & $\begin{array}{l}\text { Compare: } \\
\text { historical } \\
\text { control design } \\
(n=132)\end{array}$ & $\begin{array}{l}\text { 1) Attitudes } \\
\text { towards F\&V: } \\
\text { the attitudes } \\
\text { questionnaire } \\
\text { 2) Nutrition } \\
\text { knowledge: F\&V } \\
\text { identification } \\
\text { survey }\end{array}$ \\
\hline $\begin{array}{l}\text { McAleese et al., } \\
\text { (2007) USA [35] }\end{array}$ & $\begin{array}{l}12 \text { weeks } \\
\text { non- } \\
\text { randomised } \\
\text { controlled }\end{array}$ & $\begin{array}{l}3 \\
\text { schools } \\
(n=99)\end{array}$ & $\begin{array}{l}10-13 \text { years } \\
\text { old }\end{array}$ & - & $\begin{array}{l}\text { Garden-based } \\
\text { activities \& } \\
\text { nutrition } \\
\text { education } \\
(\mathrm{n}=45)\end{array}$ & $\begin{array}{l}\text { Compare: } \\
\text { nutrition } \\
\text { education only } \\
(n=25) \\
\text { Control: no } \\
\text { intervention } \\
(n=25)\end{array}$ & $\begin{array}{l}\text { 1) Dietary } \\
\text { intake: three 24- } \\
\text { hour food recall } \\
\text { workbooks }\end{array}$ \\
\hline
\end{tabular}

Degree of parental involvement: High parental involvement was defined as children having direct interaction with their parents that will affect the intervention outcomes such as parents participating in gardening, cultivating and cooking sessions with children at schools, otherwise it was defined as ${ }^{2}$ low parental involvement where the activities included parent newsletter distribution and take-home activities. 
Table 2

Summary on the main findings of each SGBP intervention $(n=35)$

\begin{tabular}{|c|c|c|c|c|c|c|c|c|}
\hline \multirow{2}{*}{$\begin{array}{l}\text { Author (Year) } \\
\text { and location }\end{array}$} & \multicolumn{7}{|c|}{ Components included in each intervention } & \multirow[t]{2}{*}{ Main findings } \\
\hline & $\begin{array}{l}\text { Parental } \\
\text { involvement }\end{array}$ & $\begin{array}{l}\text { School } \\
\text { gardening }\end{array}$ & $\begin{array}{l}\text { Nutrition } \\
\text { education }\end{array}$ & $\begin{array}{l}\text { Cooking } \\
\& \\
\text { kitchen } \\
\text { lesson }\end{array}$ & $\begin{array}{l}\text { Tasting } \\
\text { section }\end{array}$ & $\begin{array}{l}\text { Home } \\
\text { gardening }\end{array}$ & Others & \\
\hline \multirow{3}{*}{$\begin{array}{l}\text { Davis et al., } \\
\text { (2021) USA [1] }\end{array}$} & $\mathrm{v}$ & $\mathrm{v}$ & $\mathrm{v}$ & $\mathrm{v}$ & $\mathrm{v}$ & & Parental lessons & F intake: - \\
\hline & & & & & & & & V intake: +* \\
\hline & & & & & & & & $\begin{array}{l}\text { SSB intake } \\
\text { (reduction): - }\end{array}$ \\
\hline \multirow[t]{5}{*}{$\begin{array}{l}\text { Barnard et al., } \\
\text { (2020) USA [2] }\end{array}$} & v & $\mathrm{v}$ & $v$ & v & & & $\begin{array}{l}\text { Carrot camp; sprout } \\
\text { scout }\end{array}$ & \\
\hline & & & & & & & & Willingness to try V: - \\
\hline & & & & & & & & F intake: - \\
\hline & & & & & & & & V intake: - \\
\hline & & & & & & & & $\begin{array}{l}\text { Willing to discuss } \\
\text { what have learnt at } \\
\text { home: - }\end{array}$ \\
\hline \multirow[t]{7}{*}{$\begin{array}{l}\text { Kim et al., (2020) } \\
\text { South Korea [3] }\end{array}$} & & $\mathrm{v}$ & $v$ & v & & & & $\begin{array}{l}\text { Gardening } \\
\text { knowledge: }+\star \star \star\end{array}$ \\
\hline & & & & & & & & $\begin{array}{l}\text { Nutrition knowledge: } \\
+\star \star \star\end{array}$ \\
\hline & & & & & & & & $\begin{array}{l}\text { Preference for } \mathrm{V}: \\
+\star \star \star\end{array}$ \\
\hline & & & & & & & & 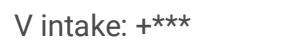 \\
\hline & & & & & & & & $\begin{array}{l}\text { Willingness to try } \\
\text { new food/ reduction } \\
\text { in Food neophobia: } \\
+* \star \star\end{array}$ \\
\hline & & & & & & & & $\begin{array}{l}\text { Dietary self-efficacy: } \\
+\star \star \star\end{array}$ \\
\hline & & & & & & & & $\begin{array}{l}\text { Outcome } \\
\text { expectancies: }+* \star \star\end{array}$ \\
\hline \multirow{7}{*}{$\begin{array}{l}\text { Schreinemachers } \\
\text { et al., }(2020) \\
\text { Nepal [4] }\end{array}$} & v & $\mathrm{v}$ & $v$ & & & $v$ & & $\begin{array}{l}\text { Food and nutritional } \\
\text { knowledge: - }\end{array}$ \\
\hline & & & & & & & & $\begin{array}{l}\text { Agriculture } \\
\text { knowledge: - }\end{array}$ \\
\hline & & & & & & & & $\begin{array}{l}\text { Attitude towards V: } \\
+^{\star}\end{array}$ \\
\hline & & & & & & & & $\begin{array}{l}\text { Healthy food } \\
\text { practise: }+\star \star \star\end{array}$ \\
\hline & & & & & & & & V intake (Oct-Dec): - \\
\hline & & & & & & & & $\begin{array}{l}\text { V intake (Jan-Mar): } \\
+^{\star}\end{array}$ \\
\hline & & & & & & & & V intake (Apr-Jun): - \\
\hline \multirow[t]{4}{*}{$\begin{array}{l}\text { Shrestha et al., } \\
\text { (2020) Nepal [5] }\end{array}$} & & $\mathrm{v}$ & & & & & & $\begin{array}{l}\text { Nutrition knowledge: } \\
\text { - }\end{array}$ \\
\hline & & & & & & & & V intake: - \\
\hline & & & & & & & & $\begin{array}{l}\text { F availability } \\
\text { (home): - }\end{array}$ \\
\hline & & & & & & & & $\begin{array}{l}\text { V availability } \\
\text { (home): - }\end{array}$ \\
\hline
\end{tabular}

v: Included 


\begin{tabular}{|c|c|c|c|c|c|c|c|c|}
\hline \multirow{2}{*}{$\begin{array}{l}\text { Author (Year) } \\
\text { and location }\end{array}$} & \multicolumn{7}{|c|}{ Components included in each intervention } & \multirow[t]{2}{*}{ Main findings } \\
\hline & $\begin{array}{l}\text { Parental } \\
\text { involvement }\end{array}$ & $\begin{array}{l}\text { School } \\
\text { gardening }\end{array}$ & $\begin{array}{l}\text { Nutrition } \\
\text { education }\end{array}$ & $\begin{array}{l}\text { Cooking } \\
\& \\
\text { kitchen } \\
\text { lesson }\end{array}$ & $\begin{array}{l}\text { Tasting } \\
\text { section }\end{array}$ & $\begin{array}{l}\text { Home } \\
\text { gardening }\end{array}$ & Others & \\
\hline \multirow{5}{*}{$\begin{array}{l}\text { van den Berg et } \\
\text { al., (2020) USA } \\
\text { [6] }\end{array}$} & v & $\mathrm{v}$ & v & & & & & $\begin{array}{l}\text { Nutrition knowledge: } \\
+\star \star \star\end{array}$ \\
\hline & & & & & & & & $\begin{array}{l}\text { Preference for V: } \\
+\star \star \star\end{array}$ \\
\hline & & & & & & & & V tasted: $+\star \star \star$ \\
\hline & & & & & & & & V intake: - \\
\hline & & & & & & & & $\begin{array}{l}\text { V availability } \\
\text { (home): - }\end{array}$ \\
\hline \multirow[t]{5}{*}{$\begin{array}{l}\text { Khan et al., } \\
\text { (2019) UK [7] }\end{array}$} & & $v$ & v & & & & $\begin{array}{l}\text { Meat-Free Monday } \\
\text { session on } \\
\text { children's healthy }\end{array}$ & $\begin{array}{l}\text { Nutrition and plant } \\
\text { science knowledge: - }\end{array}$ \\
\hline & & & & & & & $\begin{array}{l}\text { eating, physical } \\
\text { activity }\end{array}$ & $\begin{array}{l}\text { Attitude towards } \\
\text { F\&V consumption: - }\end{array}$ \\
\hline & & & & & & & & Preference for F\&V: - \\
\hline & & & & & & & & F intake: - \\
\hline & & & & & & & & V intake: - \\
\hline \multirow{6}{*}{$\begin{array}{l}\text { Landry et al. } \\
\text { (2019) USA [8] }\end{array}$} & & $\mathrm{v}$ & v & $\mathrm{v}$ & & & & Increase in cooking: \\
\hline & & & & & & & & V intake: +* \\
\hline & & & & & & & & $\begin{array}{l}\text { Dietary fibre intake: } \\
+\star \star\end{array}$ \\
\hline & & & & & & & & $\begin{array}{l}\text { Increase in } \\
\text { gardening: }\end{array}$ \\
\hline & & & & & & & & $\begin{array}{l}\text { Dietary fibre intake: } \\
+^{\star}\end{array}$ \\
\hline & & & & & & & & $\begin{array}{l}\text { Attitude and self- } \\
\text { efficacy towards } \\
\text { cooking and } \\
\text { gardening: } x\end{array}$ \\
\hline \multirow{3}{*}{$\begin{array}{l}\text { Massarani et al., } \\
\text { (2019) Rio de } \\
\text { Janeiro [9] }\end{array}$} & v & v & v & $\mathrm{v}$ & & & & F intake: - \\
\hline & & & & & & & & V intake: - \\
\hline & & & & & & & & $\begin{array}{l}\text { Ultra-processed } \\
\text { foods intake } \\
\text { (reduction): - }\end{array}$ \\
\hline \multirow{4}{*}{$\begin{array}{l}\text { Nele Huys et al., } \\
(2019) \text { Ghent } \\
{[10]}\end{array}$} & & v & v & & & & & $\begin{array}{l}\text { Nutrition knowledge: } \\
+^{*}\end{array}$ \\
\hline & & & & & & & & $\begin{array}{l}\text { Attitude towards } \\
\text { F\&V: - }\end{array}$ \\
\hline & & & & & & & & V intake: - \\
\hline & & & & & & & & Self-efficacy: - \\
\hline
\end{tabular}




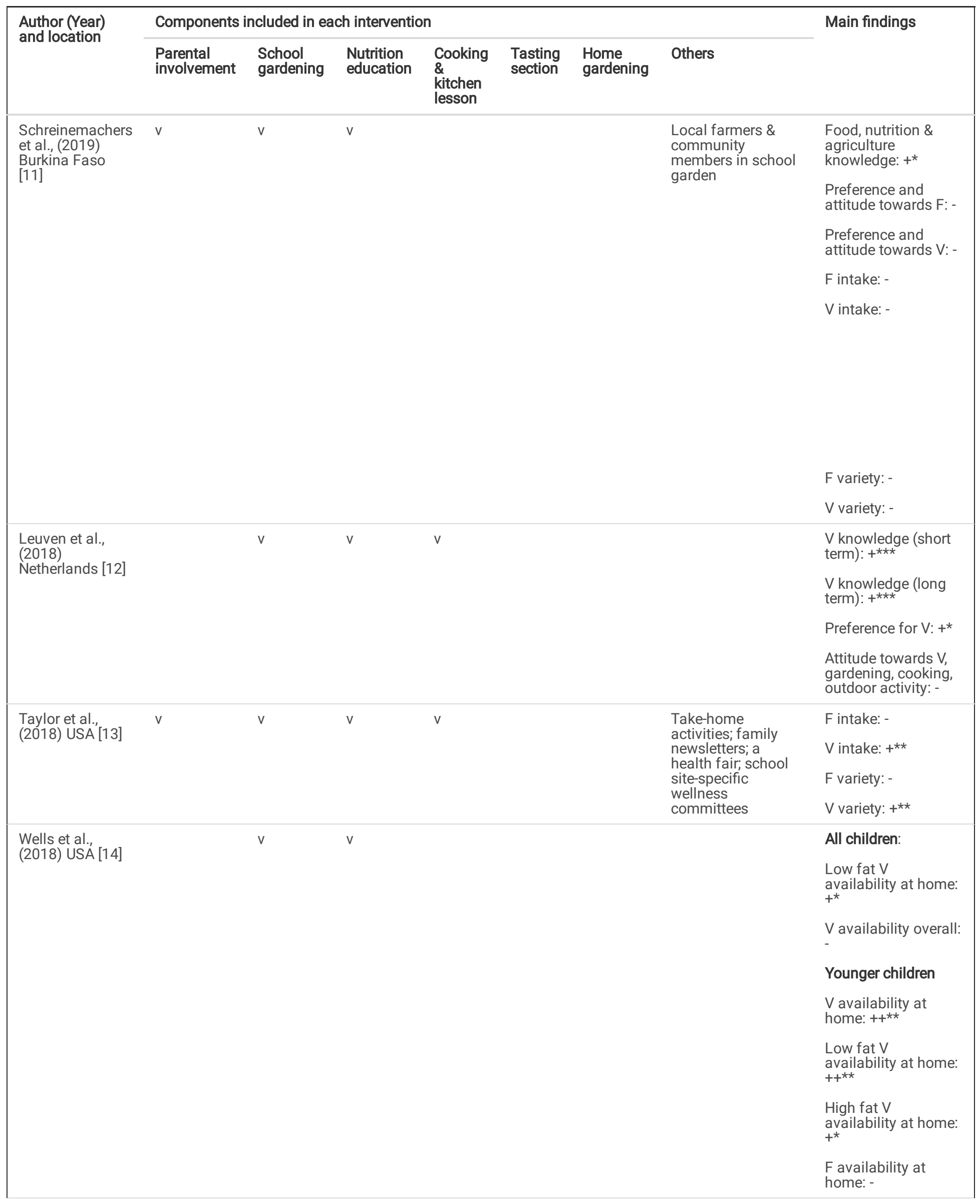




\begin{tabular}{|c|c|c|c|c|c|c|c|c|}
\hline \multirow{2}{*}{$\begin{array}{l}\text { Author (Year) } \\
\text { and location }\end{array}$} & \multicolumn{7}{|c|}{ Components included in each intervention } & \multirow[t]{2}{*}{ Main findings } \\
\hline & $\begin{array}{l}\text { Parental } \\
\text { involvement }\end{array}$ & $\begin{array}{l}\text { School } \\
\text { gardening }\end{array}$ & $\begin{array}{l}\text { Nutrition } \\
\text { education }\end{array}$ & $\begin{array}{l}\text { Cooking } \\
\& \\
\text { kitchen } \\
\text { lesson }\end{array}$ & $\begin{array}{l}\text { Tasting } \\
\text { section }\end{array}$ & $\begin{array}{l}\text { Home } \\
\text { gardening }\end{array}$ & Others & \\
\hline \multirow{3}{*}{$\begin{array}{l}\text { Gatto et al., } \\
\text { (2017) USA [15] }\end{array}$} & \multirow[t]{3}{*}{$\mathrm{v}$} & \multirow[t]{3}{*}{$\mathrm{v}$} & \multirow[t]{3}{*}{$\mathrm{v}$} & \multirow[t]{3}{*}{$\mathrm{v}$} & & & & F intake: - \\
\hline & & & & & & & & V intake: $x^{\star}$ \\
\hline & & & & & & & & $\begin{array}{l}\text { Dietary fibre intake: } \\
+^{\star}\end{array}$ \\
\hline \multirow{4}{*}{$\begin{array}{l}\text { Lee et al., (2017) } \\
\text { USA [16] }\end{array}$} & \multirow[t]{4}{*}{$\mathrm{v}$} & \multirow[t]{4}{*}{$\mathrm{v}$} & \multirow[t]{4}{*}{$\mathrm{v}$} & \multirow[t]{4}{*}{$\mathrm{v}$} & \multirow[t]{4}{*}{$\mathrm{v}$} & & & F intake: - \\
\hline & & & & & & & & V intake: - \\
\hline & & & & & & & & $\begin{array}{l}\text { V availability } \\
\text { (home): - }\end{array}$ \\
\hline & & & & & & & & $\begin{array}{l}\text { F availability } \\
\text { (home): - }\end{array}$ \\
\hline \multirow{6}{*}{$\begin{array}{l}\text { Schreinemachers } \\
\text { et al., (2017) } \\
\text { Bhutan [17] }\end{array}$} & \multirow[t]{6}{*}{$\mathrm{v}$} & \multirow[t]{6}{*}{$\mathrm{v}$} & \multirow[t]{6}{*}{ v } & & & & \multirow{6}{*}{$\begin{array}{l}\text { Promotion } \\
\text { activities: poster } \\
\text { displays, songs, } \\
\text { nutrition charts, } \\
\text { vegetable charts, } \\
\text { pledges }\end{array}$} & $\begin{array}{l}\text { Nutrition knowledge: } \\
\text { - }\end{array}$ \\
\hline & & & & & & & & $\begin{array}{l}\text { Preference towards } \\
\text { F\&V: }+^{\star}\end{array}$ \\
\hline & & & & & & & & F intake: - \\
\hline & & & & & & & & V intake: + * \\
\hline & & & & & & & & F variety: - \\
\hline & & & & & & & & V variety: - \\
\hline \multirow[t]{7}{*}{$\begin{array}{l}\text { Schreinemachers } \\
\text { et al., (2017) } \\
\text { Nepal [18] }\end{array}$} & \multirow[t]{7}{*}{$\mathrm{v}$} & \multirow[t]{7}{*}{$\mathrm{v}$} & \multirow[t]{7}{*}{$\mathrm{v}$} & & & & \multirow{7}{*}{$\begin{array}{l}\text { Poster display, } \\
\text { distribution of } \\
\text { handouts about } \\
\text { nutritious food and } \\
\text { hand washing }\end{array}$} & $\begin{array}{l}\text { Food and nutritional } \\
\text { knowledge: }+\star \star \star\end{array}$ \\
\hline & & & & & & & & Preference for $\mathrm{F}:$ +** $^{\star \star}$ \\
\hline & & & & & & & & Preference for $\mathrm{V}:{ }^{\star \star \star}$ \\
\hline & & & & & & & & F intake: - \\
\hline & & & & & & & & V intake: - \\
\hline & & & & & & & & F variety: - \\
\hline & & & & & & & & V variety: - \\
\hline
\end{tabular}




\begin{tabular}{|c|c|c|c|c|c|c|c|c|}
\hline \multirow{2}{*}{$\begin{array}{l}\text { Author (Year) } \\
\text { and location }\end{array}$} & \multicolumn{7}{|c|}{ Components included in each intervention } & \multirow[t]{2}{*}{ Main findings } \\
\hline & $\begin{array}{l}\text { Parental } \\
\text { involvement }\end{array}$ & $\begin{array}{l}\text { School } \\
\text { gardening }\end{array}$ & $\begin{array}{l}\text { Nutrition } \\
\text { education }\end{array}$ & $\begin{array}{l}\text { Cooking } \\
\& \\
\text { kitchen } \\
\text { lesson }\end{array}$ & $\begin{array}{l}\text { Tasting } \\
\text { section }\end{array}$ & $\begin{array}{l}\text { Home } \\
\text { gardening }\end{array}$ & Others & \\
\hline \multirow[t]{10}{*}{$\begin{array}{l}\text { Davis et al., } \\
\text { (2016) USA [19] }\end{array}$} & & v & $\mathrm{v}$ & $\mathrm{v}$ & & & $\begin{array}{l}\text { Local farmers' } \\
\text { market visit }\end{array}$ & $\begin{array}{l}\text { Vegetable } \\
\text { identification } \\
\text { (knowledge): +** }\end{array}$ \\
\hline & & & & & & & & $\begin{array}{l}\text { Nutrition and } \\
\text { gardening } \\
\text { knowledge: +** }\end{array}$ \\
\hline & & & & & & & & Preference for $F: x$ \\
\hline & & & & & & & & Preference for V: - \\
\hline & & & & & & & & $\begin{array}{l}\text { Willingness to try } \mathrm{F} \text { : } \\
\mathrm{x}\end{array}$ \\
\hline & & & & & & & & Willingness to try $\mathrm{V}$ : - \\
\hline & & & & & & & & $\begin{array}{l}\text { Attitude towards } \\
\text { gardening and } \\
\text { cooking: - }\end{array}$ \\
\hline & & & & & & & & $\begin{array}{l}\text { Motivation to eat FV, } \\
\text { cooking + gardening: } \\
\text { - }\end{array}$ \\
\hline & & & & & & & & $\begin{array}{l}\text { Self-efficacy to eat } \\
\text { F\&V: - }\end{array}$ \\
\hline & & & & & & & & $\underset{\star \star}{\text { Home gardening: }+}$ \\
\hline \multirow[t]{2}{*}{$\begin{array}{l}\text { Duncan et al., } \\
\text { (2015) UK [20] }\end{array}$} & & $\mathrm{v}$ & $\mathrm{v}$ & $\mathrm{v}$ & & & & $\begin{array}{l}\text { Intentions }{ }^{* * *} \text {, } \\
\text { attitudes***, } \\
\text { norms } s^{* * *} \text {, and } \\
\text { perceived } \\
\text { behavioural } \\
\text { control*** related to } \\
\text { F\&V intake: + }\end{array}$ \\
\hline & & & & & & & & F\&V intake: $+\star \star$ \\
\hline \multirow{5}{*}{$\begin{array}{l}\text { Hanbazaza et al., } \\
\text { (2015) Canada } \\
\text { [21] }\end{array}$} & & v & & & & & Healthy F\&V snack & F\&V knowledge: - \\
\hline & & & & & & & & Preference for $\mathrm{F}:+^{\star \star \star}$ \\
\hline & & & & & & & & Preference for $\mathrm{V}$ : -* \\
\hline & & & & & & & & F intake (home): - \\
\hline & & & & & & & & V intake (home): - \\
\hline \multirow[t]{6}{*}{$\begin{array}{l}\text { Sharma et al., } \\
\text { (2015) USA [22] }\end{array}$} & $v$ & $v$ & v & & & & & $\begin{array}{l}\text { Willingness to try F: } \\
+* \star\end{array}$ \\
\hline & & & & & & & & $\begin{array}{l}\text { Willingness to try } \mathrm{V} \text { : } \\
+\star \star\end{array}$ \\
\hline & & & & & & & & F\&V variety: - \\
\hline & & & & & & & & eating behaviour: - \\
\hline & & & & & & & & $\begin{array}{l}\text { F availability } \\
\text { (home): - }\end{array}$ \\
\hline & & & & & & & & $\begin{array}{l}\text { V availability } \\
\text { (home): - }\end{array}$ \\
\hline
\end{tabular}




\begin{tabular}{|c|c|c|c|c|c|c|c|c|}
\hline \multirow{2}{*}{$\begin{array}{l}\text { Author (Year) } \\
\text { and location }\end{array}$} & \multicolumn{7}{|c|}{ Components included in each intervention } & \multirow[t]{2}{*}{ Main findings } \\
\hline & $\begin{array}{l}\text { Parental } \\
\text { involvement }\end{array}$ & $\begin{array}{l}\text { School } \\
\text { gardening }\end{array}$ & $\begin{array}{l}\text { Nutrition } \\
\text { education }\end{array}$ & $\begin{array}{l}\text { Cooking } \\
\& \\
\text { kitchen } \\
\text { lesson }\end{array}$ & $\begin{array}{l}\text { Tasting } \\
\text { section }\end{array}$ & $\begin{array}{l}\text { Home } \\
\text { gardening }\end{array}$ & Others & \\
\hline \multirow{7}{*}{$\begin{array}{l}\text { Spears-Lanoix et } \\
\text { al.,(2015) USA } \\
\text { [23] }\end{array}$} & $\mathrm{v}$ & $\mathrm{v}$ & & & $v$ & & & Nutrition knowledge: \\
\hline & & & & & & & & Preference for V: +* \\
\hline & & & & & & & & Willingness to try V: - \\
\hline & & & & & & & & V intake: $+\star \star \star ~$ \\
\hline & & & & & & & & Total F\&V intake: - \\
\hline & & & & & & & & $\begin{array}{l}\text { SSB intake } \\
\text { (reduction): } x\end{array}$ \\
\hline & & & & & & & & $\begin{array}{l}\text { Home availability } \\
\text { (V): }+^{\star}\end{array}$ \\
\hline $\begin{array}{l}\text { Wells et al., } \\
\text { (2015) USA [24] }\end{array}$ & & $\mathrm{v}$ & v & & & & & $\begin{array}{l}\text { Scientific } \\
\text { knowledge: }+* * \star \text { (yet } \\
\text { the result was } \\
\text { uniformly poor) }\end{array}$ \\
\hline \multirow[t]{6}{*}{$\begin{array}{l}\text { Bontrager Yoder } \\
\text { et al., (2014) USA } \\
\text { [25] }\end{array}$} & & $\mathrm{v}$ & $\mathrm{v}$ & & & & $\begin{array}{l}\text { Harvest of the } \\
\text { Month, }\end{array}$ & 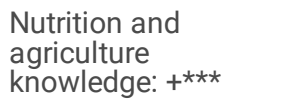 \\
\hline & & & & & & & $\begin{array}{l}\text { locally sourced } \\
\text { produce in school } \\
\text { meals }\end{array}$ & $\begin{array}{l}\text { Willingness to try } \\
\text { F\&V: +*** }\end{array}$ \\
\hline & & & & & & & & $\begin{array}{l}\text { Lunch time F\&V } \\
\text { availability: }+{ }^{\star *}\end{array}$ \\
\hline & & & & & & & & $\begin{array}{l}\text { F\&V consumption } \\
\text { among low intake: } \\
+\star \star \star\end{array}$ \\
\hline & & & & & & & & Overall F\&V intake: - \\
\hline & & & & & & & & $\begin{array}{l}\text { F\&V variety (school): } \\
+* \star \star\end{array}$ \\
\hline $\begin{array}{l}\text { Cotter et al., } \\
(2013) \text { Portugal } \\
{[26]}\end{array}$ & & $\mathrm{v}$ & $\mathrm{v}$ & & & & & $\begin{array}{l}\text { Salt intake } \\
\text { reduction: + }\end{array}$ \\
\hline \multirow{5}{*}{$\begin{array}{l}\text { Gibbs et al., } \\
\text { (2013) Australia } \\
{[27]}\end{array}$} & $\mathrm{v}$ & $\mathrm{v}$ & & $\mathrm{v}$ & & & & $\begin{array}{l}\text { Nutrition knowledge } \\
\text { (food description):- }\end{array}$ \\
\hline & & & & & & & & $\begin{array}{l}\text { Preference for } F \& V \\
\text { (if grow in garden): } \\
+^{*}\end{array}$ \\
\hline & & & & & & & & $\begin{array}{l}\text { Willingness to try } \\
\text { new food: }+^{\star}\end{array}$ \\
\hline & & & & & & & & F intake: - \\
\hline & & & & & & & & V intake: - \\
\hline \multirow[t]{6}{*}{$\begin{array}{l}\text { Gatto et al., } \\
\text { (2012) USA [28] }\end{array}$} & $v$ & $\mathrm{v}$ & $\mathrm{v}$ & v & & & $\begin{array}{l}\text { Local farmers' } \\
\text { market visit }\end{array}$ & For all \\
\hline & & & & & & & & Preference for $F: x$ \\
\hline & & & & & & & & Preference for V: - \\
\hline & & & & & & & & $\begin{array}{l}\text { For } \\
\text { obese/overweight: }\end{array}$ \\
\hline & & & & & & & & Preference for $F: x$ \\
\hline & & & & & & & & Preference for $\mathrm{V}:+^{\star \star}$ \\
\hline
\end{tabular}




\begin{tabular}{|c|c|c|c|c|c|c|c|c|}
\hline \multirow{2}{*}{$\begin{array}{l}\text { Author (Year) } \\
\text { and location }\end{array}$} & \multicolumn{7}{|c|}{ Components included in each intervention } & \multirow[t]{2}{*}{ Main findings } \\
\hline & $\begin{array}{l}\text { Parental } \\
\text { involvement }\end{array}$ & $\begin{array}{l}\text { School } \\
\text { gardening }\end{array}$ & $\begin{array}{l}\text { Nutrition } \\
\text { education }\end{array}$ & $\begin{array}{l}\text { Cooking } \\
\& \\
\text { kitchen } \\
\text { lesson }\end{array}$ & $\begin{array}{l}\text { Tasting } \\
\text { section }\end{array}$ & $\begin{array}{l}\text { Home } \\
\text { gardening }\end{array}$ & Others & \\
\hline \multirow[t]{3}{*}{$\begin{array}{l}\text { Jaenke et al., } \\
\text { (2012) Australia } \\
\text { [29] }\end{array}$} & v & v & $\mathrm{v}$ & v & & & & $\begin{array}{l}\text { Willingness to try } \mathrm{V} \text { : } \\
+\star \star \star\end{array}$ \\
\hline & & & & & & & & F intake: - \\
\hline & & & & & & & & V intake: - \\
\hline \multirow{3}{*}{$\begin{array}{l}\text { Davis et al., } \\
\text { (2011) USA [30] }\end{array}$} & $v$ & v & $v$ & $\mathrm{v}$ & & & $\begin{array}{l}\text { Local farmers' } \\
\text { market visit }\end{array}$ & \\
\hline & & & & & & & & V intake: - \\
\hline & & & & & & & & $\begin{array}{l}\text { Dietary fibre intake: } \\
+^{\star}\end{array}$ \\
\hline \multirow[t]{6}{*}{$\begin{array}{l}\text { Ratcliffe et al., } \\
\text { (2011) USA [31] }\end{array}$} & & v & $\mathrm{v}$ & & & & & $\begin{array}{l}\text { Improving } \\
\text { recognition of } \mathrm{V}:+^{\star *}\end{array}$ \\
\hline & & & & & & & & $\begin{array}{l}\text { Attitude toward \& } \\
\text { Preference for V: +* }\end{array}$ \\
\hline & & & & & & & & $\begin{array}{l}\text { Willingness to try } \mathrm{V} \text { : } \\
+\star \star \star\end{array}$ \\
\hline & & & & & & & & V intake (school): + * \\
\hline & & & & & & & & V intake (home): - \\
\hline & & & & & & & & V variety: $+\star \star \star ~$ \\
\hline \multirow{5}{*}{$\begin{array}{l}\text { Morgan et al. } \\
(2010) \text { Australia } \\
{[32]}\end{array}$} & v & v & v & v & & & & F\&V knowledge: $+\star \star \star ~$ \\
\hline & & & & & & & & $\begin{array}{l}\text { Taste rating for } \mathrm{V} \\
\text { (preference): }+* \star \star \star\end{array}$ \\
\hline & & & & & & & & $\begin{array}{l}\text { Willingness to try } \mathrm{V} \text { : } \\
+\star \star \star\end{array}$ \\
\hline & & & & & & & & F intake: - \\
\hline & & & & & & & & V intake: - \\
\hline \multirow[t]{5}{*}{$\begin{array}{l}\text { Parmer et al., } \\
\text { (2009) USA [33] }\end{array}$} & & v & $v$ & & & & & $\begin{array}{l}\text { Nutrition knowledge: } \\
+\star \star\end{array}$ \\
\hline & & & & & & & & $\begin{array}{l}\text { Rating of tasted } \\
\text { F\&V: + ** }\end{array}$ \\
\hline & & & & & & & & $\begin{array}{l}\text { Willingness to try } \\
\text { F\&V: - }\end{array}$ \\
\hline & & & & & & & & V choice: $+*$ \\
\hline & & & & & & & & V intake: +* $^{*}$ \\
\hline
\end{tabular}




\begin{tabular}{|c|c|c|c|c|c|c|c|c|}
\hline \multirow{2}{*}{$\begin{array}{l}\text { Author (Year) } \\
\text { and location }\end{array}$} & \multicolumn{7}{|c|}{ Components included in each intervention } & \multirow[t]{2}{*}{ Main findings } \\
\hline & $\begin{array}{l}\text { Parental } \\
\text { involvement }\end{array}$ & $\begin{array}{l}\text { School } \\
\text { gardening }\end{array}$ & $\begin{array}{l}\text { Nutrition } \\
\text { education }\end{array}$ & $\begin{array}{l}\text { Cooking } \\
\& \\
\text { kitchen } \\
\text { lesson }\end{array}$ & $\begin{array}{l}\text { Tasting } \\
\text { section }\end{array}$ & $\begin{array}{l}\text { Home } \\
\text { gardening }\end{array}$ & Others & \\
\hline \multirow[t]{5}{*}{$\begin{array}{l}\text { Somerset et al., } \\
\text { (2009) Australia } \\
\text { [34] }\end{array}$} & & v & & & & & $\begin{array}{l}\text { Funding of a } \\
\text { teacher coordinator } \\
\text { (facilitate }\end{array}$ & $\begin{array}{l}\text { Nutrition knowledge } \\
\text { (ability to identify } \\
\text { F\&V): +*}\end{array}$ \\
\hline & & & & & & & $\begin{array}{l}\text { garden activities } \\
\text { into curriculum) }\end{array}$ & $\begin{array}{l}\text { Interest in trying new } \\
\text { food: } x\end{array}$ \\
\hline & & & & & & & & $\begin{array}{l}\text { Preference towards } \\
\text { F: - }\end{array}$ \\
\hline & & & & & & & & $\begin{array}{l}\text { Preference towards } \\
\text { V: }+^{*}\end{array}$ \\
\hline & & & & & & & & Dietary self-efficacy: \\
\hline \multirow{5}{*}{$\begin{array}{l}\text { McAleese et al., } \\
\text { (2007) USA [35] }\end{array}$} & & v & v & & & & & F intake: $++\star \star \star$ \\
\hline & & & & & & & & V intake: $++\star \star *$ \\
\hline & & & & & & & & Vitamin A intake: +** \\
\hline & & & & & & & & $\begin{array}{l}\text { Vitamin C intake: } \\
++^{\star}\end{array}$ \\
\hline & & & & & & & & Fibre intake: + ** \\
\hline
\end{tabular}

+: Positive finding

++: Positive findings with more significant findings shown (compare within the same study)

$\therefore$ No change/ negative

$\mathrm{x}$ : Negative findings

$*: p<0.05 ; * *: p<0.01 ; * * *: p<0.001$

\section{Major findings}

Due to no significant difference in the outcomes between the intervention with and without parental involvement, findings were combined and analysed all at once (Fig. 2). It is noticed that a non-significant increase is regarded as no change in effectiveness as reported by the studies.

\section{Dietary practices and food consumption}

Children's F\&V intake was the most studied outcome $(n=26) .58 \%$ (15 out of 26$)$ of the studies failed to find a significant improvement in vegetable consumption with the intervention $[2,5-7,9-11,15,16,18,25,27,29,30,32]$, while this figure raised to $88 \%$ (15 out of 17$)$ for children's fruit consumption $[1,2,7,9,11,13,15-18,23,27,29,30,32]$. These findings reflect that school garden-based programmes might not be effective in improving F\&V consumption among children. Several studies also reported the impact of SGBP on increased dietary fibre ( $\mathrm{n}=4)$, and Vitamin A and Vitamin C ( $\mathrm{n}=1)$ intake $[8,15,30,35]$. Interestingly, a study conducted on the Portuguese population showed a promising effect of SGBP in reducing students' salt intake [26]. Some studies also investigated the intervention effect in reducing sugar-sweetened beverages $(S S B)(n=2)$ and ultra-processed food consumption ( $n=1)$, with all showing neutral effects $[1,9,23]$.

\section{Nutrition, gardening, agriculture and science-related knowledge}

Nutrition, gardening, agriculture and science-related knowledge was the second most studied outcome $(n=20)$. The duration of the intervention ranged from 9 weeks to 2.5 years among the students aged 6 to 17 years old. Most of the studies have demonstrated statistically significant improvement in children's nutritional knowledge after the intervention ( $n=14 / 20)[3,6,10-12,14,18,19,23,25,31-34]$.

\section{Attitudes and behaviours towards fruits and vegetables}

Two third of the reported studies showed a statistically significant improvement in their attitudes and behaviours towards vegetables after the intervention ( $n=13 / 19)[3,4,6,12,17,18,20,23,27,31-34]$. However, the effect of school garden-based intervention in improving attitudes, preferences and taste ratings towards fruits $(n=12)$ remained inconclusive, as none of the groups (neither "effective" nor the "no change" group) 
dominated each other (positive impact: $n=6 / 12[17,18,20,21,27,33]$; no change: $n=6 / 12$ [7, 10, 11, 19, 28, 34]). In addition, SGBP failed to improve the attitudes and preferences towards gardening, cooking and science in all three studies these outcomes were investigated [8, 12, 19].

Numerous studies have reported the impacts of SGBP on children's willingness to consume fruits $(n=9)$ and vegetables ( $n=12)$. The duration of the intervention ranged from 12 weeks to 4 years. Most of the studies $(n=7 / 12)$ reported statistically significant improvement in their willingness to consume vegetables at the post-intervention level $[3,22,25,27,29,31,32]$. Only a small number of studies $(n=5 / 12)$ reported no significant postintervention change $[2,19,23,33,34]$. However, with respect to the children's willingness to consume fruits, there is no obvious improvement observed $(n=5 / 9)[2,3,19,22,25,27,31,33,34]$.

\section{Other outcomes of interest}

Four studies reported on the dietary self-efficacy in children, which refers to children's self-belief in their food knowledge level and the selfconfidence to purchase, plan, prepare and cook food, as well as to make appropriate food decisions to achieve higher nutritional value [36]. Most of the studies reported no significant improvement in dietary self-efficacy $(n=3 / 4)[3,10,19,34]$. In addition, a small number of studies investigated the impact on the variety of fruits $(n=6)$ and vegetables $(n=7)$ consumed, with the majority failing to demonstrate a significant improvement $(n=$ $5 / 6 ; n=4 / 7$, respectively) $[11,13,17,18,22,25,31]$.

Seven studies reported the impact of SGBP on home F\&V availability and consumption $[5,6,14,16,21,22,31]$. Only one study from the US reported positive findings on home vegetable availability [23]. In addition, one study from the US investigated the effect on school F\&V availability with a positive finding observed [25]

\section{Quality appraisal of included studies}

Almost half of the studies included had a low risk of bias with the remaining rated unclear risk (neutral). No study included had a high risk of bias. Categories that were commonly rated as weak (i.e. with more than half of the studies rated the high risk of bias) were statistical analysis, blinding and withdrawal description (Fig. 3). Most of the studies $(n=33 / 35)$ failed to apply appropriate statistical analysis, studies rated as low risk of bias in this category were able to address the confounding factors as well as the application of intention to treat analysis. The majority of the studies (n $=32 / 35$ ) failed to describe the allocation concealment or blinding of researchers, participants, or data collectors. In addition, a large proportion of studies did not describe the method of handling withdrawals $(n=24 / 35)$, including the follow-up method and withdrawal reasons. Details of the quality appraisal for each study are provided in the Supplementary Table 2.

\section{Discussion}

\section{Are SGBPs effective in improving diet-related knowledge, attitudes and practices among school children?}

Despite the proposal of the knowledge, attitudes and behaviour communication model by Contento et al. (1992), which suggested a linear positive association between the three components that potentially influence the "practice" of such behaviour, recent research argued that such a relationship is far more reciprocal and dynamic [36, 37]. It is thus essential to understand the impacts of SGBP in addressing such association to maximise its intervention effect towards improving children F\&V intake to alleviate childhood malnutrition. In this review, the findings generated from the 35 studies included indicated that school garden-based programmes were effective in increasing diet and nutritional knowledge, as well as promoting positive attitudes and behaviours (acceptability) towards vegetables among the school children, however, no significant improvement was found in their dietary practices such as F\&V consumption and dietary diversity.

\section{Positive impacts on food, nutrition, gardening and science-related knowledge}

In this review, SGBP has shown promising effects in improving children's knowledge of food, nutrition, gardening and science (Fig. 2). Active participation in school gardening activities in combination with in-class food and nutrition curriculum has strengthened not only children's horticulture skills but also children's declarative knowledge (what is a healthy diet), procedural knowledge (how to achieve a healthy diet) and conditional knowledge (when and why healthy diet) [12,36,37]. Children thus have a higher potential and ability to make better and healthier food choices. In addition, children have demonstrated a greater ability to identify unfamiliar food, as less typical vegetables are intentionally emphasized in class during the intervention and incorporated into cooking activities and recipes [8]. The "seed to mouth" nature of the cooking and gardening programme, where children eat what they have grown in the school garden, also explains why children are more likely to recognize the types of crops they have consumed [8].

\section{Positive impacts on promoting attitudes and acceptability towards foods}

SGBP may encourage children's attitudes and acceptability to consume new food and reduce food neophobia via increasing their F\&V exposure. Food neophobia refers to the reluctance to eat and try novel food, often acts as a barrier to promote F\&V preference and consumption among children and contribute to the development of unhealthful food habits [38-40]. In this review, SGBP was successful in improving children's willingness to consume F\&V. The direct involvements in growing and cooking own garden produces were associated with an increase in children

Page 24/34 
self-reported willingness to consume new food [27, 32]. Additionally, the nature of the kitchen gardening programme, where children are encouraged to freely taste and share self-prepared meals with each other during kitchen class with no pressure to eat, has created a favourable social environment for children to try unfamiliar food and potentially reduce their food neophobic rate. It is worth noticing that a study conducted by Morgan et al., 2010 reported an increase in willingness and preference towards vegetables not only on the vegetables grown in the school garden, but also those in general, suggesting the intervention was successful in exerting influence beyond scope of the school garden, and even extended to those children did not directly expose to [29].

Interestingly, improvements were observed in children's attitudes and acceptability towards vegetables, but not for the fruits. Previous evidence suggested that being actively involved in the food production and preparation process may exert positive influences on foods that are particularly hard to change preference towards [41,42]. The hands-on learning experience provided by SGBP offers children regular positive exposure to vegetables. Through direct experience, for instance, crops growing, harvesting as well as food preparation, children increase familiarity with vegetables, and thus more likely to positively accept and improve taste preference towards such food [43-45]. This might also explain the insignificant intervention effect on improving children's attitude towards fruits as the crops grown in the interventions were dominated by vegetable species, children thus had comparatively fewer opportunities to interact with fruits and thus lowering the chance to increase their acceptability towards such food. It is also worth noticing that no observable influence was found regarding participants' age and intervention duration in shaping children's attitude towards F\&V as successful interventions were reported to have lasted from 12 weeks to 2.5 years with participants aged from 4 to 15 years old.

\section{Limited impacts on dietary practice and food consumption}

Intervention effects on improving F\&V dietary intake remain inconclusive due to the mixed results generated from the studies. Furthermore, no influence was found regarding participants' age and intervention duration in affecting children's F\&V consumption. F\&V knowledge is one of the most important determinants of their consumption [3, 19,46-48]. This is also supported by the social cognitive theory as augmentation in foodand nutrition-related knowledge with the acquisition of horticultural skills could increase the behavioural capacity regarding F\&V intake [49, 50]. Knowledge, attitudes, taste preferences and acceptability are often being described as one of the strongest predictors for future F\&V intake [8, 19, 21]. However, in this review, our finding demonstrated that SGBPs improvement in such predictors may not be sufficient enough to translate into an actual increase in F\&V uptake.

The weak intervention effect on dietary practices found in this review denotes SGBP might fail to address other determinants that might exert a greater influence on children's F\&V consumption. This includes school and home F\&V availability, parental food habits and feeding practices, the level of perceived behaviour control (i.e., ease of increasing F\&V intake) and dietary self-efficacy level (i.e., self-confidence in being able to increase F\&V intake) among individuals, as well as the peers' influences [51-54]. Children spend most of the time learning and working as a team at school, they are more likely to follow the group perspective and consider less about their own attitudes and beliefs [55]. In other words, when the peers are more health-conscious, individuals are more likely to consume more healthy food such as F\&V regardless of their own attitude towards such foods $[55,56]$.

Nonetheless, studies with positive findings on improving dietary practice found that SGBP was helpful in building a "personal connection" between children and the crops they have grown through direct experience on crop planting and nurturing. The "garden-grown" nature also adds an extra value to the F\&V, children are thus more inclined to try and consume those healthful foods [8,34]. An increase in school F\&V availability during intervention also acts as a drive-in promoting children's F\&V consumption [31]. Moreover, SGBP can exert its influence beyond the school setting, as it is found higher varieties of vegetables not cultivated from the school garden were consumed [31]. However, the studies with positive findings may be prone to bias. For instance, the increase in vegetable intake observed by Parmer et al. 2009 [33] was predominantly male participants. The positive result from McAleese et al. [35] might be prone to measurement error, as a one-time 24-hour food recall was used. The use of one-time 24hour recall to capture food intake might fail to obtain representable data due to the varied eating habits among individuals [17, 18]. Improvements in vegetable consumption observed from Ratcliffe et al. [31] and McAleese et al. [35] were mainly due to the reduced intake in the control group, thus implying a small improvement in the intervention group.

\section{Other measure outcomes - home food environment}

In this review, there was a small number of studies that investigated the intervention impact on children's home $F \& V$ availability $(n=6)$ and consumption $(n=2)$. Home F\&V availability is known to be a crucial determinant in affecting children's food choices. If F\&V is not readily available at home, it is difficult for the children to transfer what they have learnt from the intervention into everyday life [17, 18]. However, in this study, we did not observe any significant impacts of SGBP on home F\&V availability and consumption. The potential explanations account for the neutral effect of SGBP in modifying children's home F\&V consumption include an increase in age (lessoning effect on parental influences), family influences, unappealing presentation of F\&V in home meal, low home food security, comparatively higher cost of F\&V, limited accessibility to F\&V within community and media influences [36-38]. With respect to home F\&V availability, the authors speculated that the failure of increasing the home F\&V availability may be due to the low parental participation or response rate, which subsequently hinders the children's F\&V uptake at home, as parents remain to be the main nutrition gatekeeper [6]. Since children spend a significant amount of time at home, they are more likely to enjoy and consume food that their parents enjoy, or vice versa, and prefer foods that are readily accessible and available in the home environment [39, 40].

Page 25/34 
Thus, it is important to improve parents' KAP on diet and nutrition as well as the home F\&V availability and accessibility to improve children's food choices. Besides, it may be due to other determinants such as socioeconomic status and cultural influences, as well as the accessibility and availability of such foods within the neighbourhood [41-43].

\section{The type, the extent and the length of SGBP}

The nature of SGBP plays a key role in determining the success of the intervention. It is observed that all SGBP included in this review provided hand-on gardening experience, with most offered alongside nutrition education $(n=30)$, and cooking lesson $(n=16)$, which were shown to be effective in improving children's knowledge, attitudes, and acceptability towards healthy eating practises. Other activities, including tasting section $(n=3)$, local farmers' market visit $(n=3)$, local farmers and community members participating in gardening $(n=1)$, locally sourced produce included in school meals $(n=1)$ and other promotional activities such as poster display $(n=2)$, nutrition handout distribution ( $n=1)$, healthy F\&V snack programme $(n=1)$, Carrot camp $(n=1)$, harvest of the month $(n=1)$, meat-free Monday section $(n=1)$ and take-home activities $(n=1)$, were also investigated in some studies with unclear additional effect on improving children's KAP [44]. The effectiveness and the need for such components are thus questionable and worth to be further investigated.

Previous evidence has shown school gardening interventions that succeed in enhancing nutrition knowledge lasted for a minimum of 17 weeks [10]. This review, however, reveals a minimum of 9 to 12 weeks of intervention is sufficient to observe a significant improvement in nutritional knowledge among school children aged 8-14 years old, particularly from the US population, implying shorter intervention is as effective as a longer intervention in improving in such outcome. A probable explanation is those interventions with shorter duration might have a similar quality, intensity, and programme exposure as those with longer duration [45]. In addition, interventions that succeed in reducing children food neophobic rate in this review was mostly conducted within 5 months to 1 year, implying short intervention favours the improvement in children's food acceptability whereas long intervention might demotivate children and potentially reduce the intervention effect, as SGBP turns from initially a "novel experience" into a "mainstream activity" [46].

\section{Does parent participation benefit the school-garden based programmes?}

This review reveals that parental involvement in SGBP did not exert a significant effect on improving children's KAP towards healthy eating. Previous evidence has recognised the importance of establishing a good partnership between school, family and community for successful education programmes [36-39]. Parents play a fundamental role in the development and achievements of students. According to social learning theory, children learn and model from the behaviours of others through observation [40] As children spend a substantial amount of time with parents, they are thus hugely influenced by parents' words, behaviours and beliefs [41-43]. An effective parental involvement, including active faceto-face engagement in the programme and frequent interaction with children during the intervention, may encourage children to be more actively engaged in school activities which might potentially lead to positive intervention outcomes.

Despite the evidence has demonstrated the vital role of parents towards intervention success, the insignificant finding could be speculated by the low degree of parental involvement activities with low parents' participation rate in intervention. Studies conducted by Jaenke et al. (2012), Lee et al. (2017), Taylor et al. (2018) and Massarani et al. (2019) only included regular parent newsletter distribution as their main parents' engagement activities, as newsletter could only serve as an informed purpose, parent-children interactions were hence limited [9, 13, 16, 29]. In addition, parents often have inflexible and overwhelming work schedules with multiple responsibilities in charge, resulting in limited time dedicated to each responsibility and hence a low parents' participation in school activities is expected [44]. Therefore, it is understandable that no significant parental effect was observed. Moreover, some of the studies did not describe the parental involvement activities in detail (e.g. the number of parents involved and the response rate were absent), which make it difficult to assess its impacts on the effectiveness of the interventions. Future SGBP, hence, should measure the degree of parental engagement, if possible, examine what constitutes effective parental involvement and identify effective strategies to promote and maximise parental interaction with children during the intervention. To encourage more parental engagement, it requires better cooperation between the school and parents. It is suggested to schedule meetings and activities on multiple occasions to match parents' varying schedules and be flexible in accommodating parents and families in the school programmes such as providing incentives, food or refreshments, and free transportation to minimise barriers and create an enabling environment for parents' participation [45].

\section{SGBP in developed and developing countries}

Six studies included are from either developing or least developed countries with weak intervention effects observed. Improvements were only found in the children's preferences and attitudes towards vegetables with the majority of the studies failing to improve nutrition, gardening and agriculture-related knowledge, with nearly all reported no significant improvement observed in F\&V intake. The parental influences on shaping children's dietary behaviour were weak possibly due to their comparatively low education level $[4,17]$. With limited knowledge and awareness of nutrition and healthy diet, parents from less developed countries might be less likely to make healthier food choices for their children, family modelling impact is thus reduced.

Moreover, inadequate school resources may be one of the reasons for the insignificant gardening effects. Due to the limited number of teachers and fewer classroom materials provided, teachers from low-income countries are already struggling with completing their high amount of workload. Schreinemachers et al. (2019) reported that teaching in Burkina Faso is difficult as every teacher is averagely responsible for 45 primary 
schoolchildren, requesting them to take on extra responsibilities and time for the implementation of an unessential gardening intervention are thus extremely hard [11]. The intervention effects might consequently be undermined as teachers are less likely to deliver the intervention programme due to the constraints of time and resources. Furthermore, the seasonal supply of vegetables also lowers the intervention effect. Due to the limited water supply in the dry season, vegetables are reported to be available for only 3 to 4 months, effect on improving children's preference and intake of F\&V are therefore reduced due to the limited availability and accessibility of F\&V [11]. Besides, it is known that most school children from developing countries already participated in agricultural activities at home, garden-based intervention might be more appealing to those from developed countries where children lack nature experience and outdoor activities at school [11].

\section{Strengths and limitations}

One of the strengths of this review is that a comprehensive literature search was performed from five different databases to adequately identify most of the literature related to this topic, and potentially reduced the selection bias [36]. A robust review method was used, as two reviewers were involved to determine the inclusion and exclusion of studies independently. In addition, based on the Quality Criteria Checklist from the Academy of Nutrition and Dietetics (2016), none of the included studies was rated as high risk of bias, this demonstrates the high quality of the included studies.

There are a few limitations that cannot be ignored. The use of various measurement tools to assess the outcome measures increases the complexity of interpretation when comparing between studies. Heterogeneity of the intervention components, sample sizes, study designs and outcome measures between studies implied that the synthesis of the meta-analysis was not possible. Therefore, a single summary estimate of the impacts of SGBP failed to be generated [37]. Moreover, all SGBPs included were multifaceted with varying degrees of cooking, gardening, and nutrition components. It is thus difficult to assess which aspect of these components and how intense (or what dose) these components were most likely to be associated with positive outcomes on children's diet and nutrition-related KAP. Besides, most of the SGBP did not consider the variation of teacher experience and motivation, which are some of the determinants for programme effectiveness. The use of varying teaching approaches and enthusiasm in curriculum delivery may influence students' learning outcomes, and potentially determine the success of the intervention [29, 38]. In addition, the review might be prone to selection bias as only studies written in English were included. Furthermore, evidence was based on studies in which the participants were predominantly US and European populations with only a small amount from Asian or other countries, therefore the findings generated may not be generalizable and transferable to the other populations.

\section{Recommendations on future SGBP}

To strengthen the impact of SGBP in promoting children's KAP as observed from most of the successful interventions, integrated SGBP which include multiple or additional components such as cooking aspects and nutritional education activities are encouraged to maximise the intervention effect. Future SGBP should also adopt a multi-level approach, which covers the school, home and community environment to maximise the scope and therefore the impact of intervention [39]. In addition, to further promote F\&V consumption among children at school, it is recommended to incorporate food service into SGBP intervention. Potential ways include setting up a school salad bar in the cafeteria using the crops grown from SGBP or utilizing the produces to supplement the food in the cafeteria to increase the accessibility and F\&V availability at school. Future SGBP should also consider building relationships or partnerships with the local farmers or community gardening or, promoting or providing the students and their families the locally grown produce so as to maximise the exposure to F\&V and the potential to promote such intake.

Despite insignificant effects observed in this review, home food availability as well as parental influence, particularly parents' feeding practice and KAP on healthy diet and nutrition, have been consistently reported to be associated with increased F\&V intake in the child. Thus, future studies should investigate the effective strategies to improve parental participation and involvement to strengthen the impact of SGBP in improving F\&V consumption. In addition, it is essential for parents to acknowledge their key role in shaping children's eating habits. Future SGBP should offer more parental lessons and provide parents with the knowledge and tools to improve children's eating behaviours. This includes 1) offering practical advice on fostering children's preferences towards healthier food options and increasing their willingness to consume unfamiliar food; 2 ) understanding the negative impact of coercive feeding practice and providing alternative options; 3 ) helping parents to establish a good parental role model; 4) educating the importance of not overfeeding their children and not forcing them to finish the meal when full [40]. Furthermore, more focus should be placed on investigating the long-term impact and the sustainability of the future SGBP.

\section{Conclusion}

School garden-based programmes have shown beneficial effects on children's knowledge of diet and nutrition as well as their attitudes and acceptability towards vegetables, but not for fruits. However, no convincing evidence supports its beneficial effects on the children's dietary practices, including the actual consumption of fruits and vegetables and the diversity of the diets. These findings suggest that other environmental factors may have played a more critical role in affecting children's food choices and dietary practices. Previous evidence reported that parents play a vital role in shaping children's food choices and dietary habits. However, the insignificant parental effect observed in this review might be due to the unenthusiastic parent participation rate or low activity intensity. Therefore, more measures are needed to be taken to encourage parental engagement so as to maximise the intervention effect. Future SGBP is suggested to use a combined multidisciplinary and multi-level approach 
targeting the children, parents and community to effectively promote healthy eating among the children and prevent childhood obesity. This would ensure that the interventions tackle individual intake as well as the factors affecting the social, family and school environment.

\section{Abbreviations}

F\&V; fruits and vegetables, KAP; knowledge, attitudes, and practices, SGBP; school garden-based programme(s), UK; United Kingdom, US; United States, WHO; World Health Organization

\section{Declarations}

\section{Acknowledgements}

The authors would like to acknowledge funding support from the UK Biotechnology and Biological Sciences Research Council (BBSRC).

\section{Funding}

This research was funded by UK Biotechnology and Biological Sciences Research Council (BBSRC) (Grant number: BB/T008989/1), with a project title of "Addressing micronutrient deficiencies associated with the double burden of childhood malnutrition in China, a combined food system framework".

\section{Author's contribution}

CLC and PYT conducted searches, screening, quality assessment and data extraction; CLC wrote the first draft of the manuscript; PYT and YYG edited and revised the manuscript; all the authors read and approved the final manuscript.

\section{Availability of data and materials}

The datasets used and/or analysed during the current study are available from the corresponding author on reasonable request.

\section{Ethics approval and consent to participate}

Not applicable.

\section{Consent for publication}

Not applicable.

\section{Competing interests}

The authors declare that they have no competing interests.

\section{References}

1. Popkin BM, Corvalan C, Grummer-Strawn LM. Dynamics of the double burden of malnutrition and the changing nutrition reality. The Lancet. 2020;395(10217):65-74.

2. Abarca-Gómez L, Abdeen ZA, Hamid ZA, Abu-Rmeileh NM, Acosta-Cazares B, Acuin C, Adams RJ, Aekplakorn W, Afsana K, Aguilar-Salinas CA. Worldwide trends in body-mass index, underweight, overweight, and obesity from 1975 to 2016: a pooled analysis of 2416 population-based measurement studies in 128. 9 million children, adolescents, and adults. The Lancet. 2017;390(10113):2627-2642.

3. Hawkes C. Global nutrition report 2017: Nourishing the SDGs. Development Initiatives. 2017.

4. Yoder ABB, Liebhart JL, McCarty DJ, Meinen A, Schoeller D, Vargas C, LaRowe T. Farm to elementary school programming increases access to fruits and vegetables and increases their consumption among those with low intake. Journal of Nutrition Education and Behavior. 2014;46(5):341-349.

5. World Cancer Research Fund. Diet, Nutrition, Physical Activity and Cancer: a Global Perspective. A summary of the Third Expert Report. 2012. Retrieved from https://www.wcrf.org/wp-content/uploads/2021/02/Summary-of-Third-Expert-Report-2018.pdf 
6. World Health Organization. Healthy diet - Fact sheets. 2020. Retrieved from https://www.who.int/news-room/fact-sheets/detail/healthy-diet

7. Howarth NC, Saltzman E, Roberts SB. Dietary fiber and weight regulation. Nutrition Reviews. 2001;59(5):129-139.

8. Rasmussen M, Krølner R, Klepp K-I, Lytle L, Brug J, Bere E, Due P. Determinants of fruit and vegetable consumption among children and adolescents: a review of the literature. Part l: quantitative studies. International Journal of Behavioral Nutrition and physical activity. 2006;3(1):1-19.

9. Ventura E, Davis J, Byrd-Williams C, Alexander K, McClain A, Lane CJ, Spruijt-Metz D, Weigensberg M, Goran M. Reduction in risk factors for type 2 diabetes mellitus in response to a low-sugar, high-fiber dietary intervention in overweight Latino adolescents. Archives of Pediatrics \& Adolescent Medicine. 2009; 163(4):320-327.

10. Ventura EE, Davis JN, Alexander KE, Shaibi GQ, Lee W, Byrd-Williams CE, Toledo-Corral CM, Lane CJ, Kelly LA, Weigensberg MJ. Dietary intake and the metabolic syndrome in overweight Latino children. Journal of the American Dietetic Association. 2008;108(8):1355-1359.

11. Miller SJ, Batra A, Shearrer G, House B, Cook L, Pont S, Goran M, Davis J. Dietary fibre linked to decreased inflammation in overweight minority youth. Pediatric Obesity. 2016;11(1):33-39.

12. Lynch C, Kristjansdottir AG, Te Velde SJ, Lien N, Roos E, Thorsdottir I, Krawinkel M, de Almeida MDV, Papadaki A, Ribic CH. Fruit and vegetable consumption in a sample of 11-year-old children in ten European countries-the PRO GREENS cross-sectional survey. Public Health Nutrition. 2014;17(11):2436-2444.

13. Green L, Kreuter M. The precede-proceed model. Health promotion planning: an educational approach. 3rd ed. Mountain View (CA): Mayfield Publishing Company; 1999. p. 32-43.

14. Bere E, Klepp K-I. Changes in accessibility and preferences predict children's future fruit and vegetable intake. International Journal of Behavioral Nutrition and Physical Activity. 2005;2(1):1-8.

15. Singer MR, Moore LL, Garrahie EJ, Ellison RC. The tracking of nutrient intake in young children: the Framingham Children's Study. American Journal of Public Health. 1995;85(12):1673-1677.

16. Resnicow K, Davis-Hearn M, Smith M, Baranowski T, Lin LS, Baranowski J, Doyle C, Wang DT. Social-cognitive predictors of fruit and vegetable intake in children. Health Psychology. 1997;16(3):272.

17. Skinner JD, Carruth BR, Bounds W, Ziegler PJ. Children's food preferences: a longitudinal analysis. Journal of the American Dietetic Association. 2002;102(11):1638-1647.

18. Thompson VJ, Bachman CM, Baranowski T, Cullen KW. Self-efficacy and norm measures for lunch fruit and vegetable consumption are reliable and valid among fifth grade students. Journal of Nutrition Education and Behavior. 2007;39(1):2-7.

19. Hanbazaza MA, Triador L, Ball GD, Farmer A, Maximova K, Nation AF, Willows ND. The impact of school gardening on Cree children's knowledge and attitudes toward vegetables and fruit. Canadian Journal of Dietetic Practice and Research. 2015;76(3):133-139.

20. Ratcliffe MM, Merrigan KA, Rogers BL, Goldberg JP. The effects of school garden experiences on middle school-aged students' knowledge, attitudes, and behaviors associated with vegetable consumption. Health promotion practice. 2011;12(1):36-43.

21. Evans A, Ranjit N, Rutledge R, Medina J, Jennings R, Smiley A, Stigler M, Hoelscher D. Exposure to multiple components of a garden-based intervention for middle school students increases fruit and vegetable consumption. Health Promotion Practice. 2012;13(5):608-616.

22. Story M, Kaphingst KM, Robinson-O'Brien R, Glanz K. Creating healthy food and eating environments: policy and environmental approaches. Annu Rev Public Health. 2008;29:253-272.

23. Christian MS, Evans CE, Nykjaer C, Hancock N, Cade JE. Evaluation of the impact of a school gardening intervention on children's fruit and vegetable intake: a randomised controlled trial. International Journal of Behavioral Nutrition and Physical Activity. 2014;11(1):1-15.

24. Schreinemachers P, Bhattarai DR, Subedi GD, Acharya TP, Chen H-p, Yang R-y, Kashichhawa NK, Dhungana U, Luther GC, Mecozzi M. Impact of school gardens in Nepal: a cluster randomised controlled trial. Journal of Development Effectiveness. 2017;9(3):329-343.

25. Schreinemachers P, Rai BB, Dorji D, Chen H-p, Dukpa T, Thinley N, Sherpa PL, Yang R-Y. School gardening in Bhutan: Evaluating outcomes and impact. Food Security. 2017;9(3):635-648.

26. Evans CE, Christian MS, Cleghorn CL, Greenwood DC, Cade JE. Systematic review and meta-analysis of school-based interventions to improve daily fruit and vegetable intake in children aged 5 to 12 y. The American Journal of Clinical Nutrition. 2012;96(4):889-901.

27. Magistrelli A, Chezem JC. Effect of ground cinnamon on postprandial blood glucose concentration in normal-weight and obese adults. Journal of the Academy of Nutrition and Dietetics. 2012;112:1806-1809. doi:10.1016/j.jand.2012.07.037

28. Davis JN, Pérez A, Asigbee FM, Landry MJ, Vandyousefi S, Ghaddar R, Hoover A, Jeans M, Nikah K, Fischer B. School-based gardening, cooking and nutrition intervention increased vegetable intake but did not reduce BMI: Texas sprouts-a cluster randomized controlled trial. International Journal of Behavioral Nutrition and Physical Activity. 2021;18(1):1-14.

29. Barnard M, Mann G, Green E, Tkachuck E, Knight K. Evaluation of a Comprehensive Farm-to-School Program: Parent and Teacher Perspectives. Journal of Hunger \& Environmental Nutrition. 2020;15(6):794-808.

30. Kim S-O, Park S. Garden-based integrated intervention for improving children's eating behavior for vegetables. International Journal of Environmental Research and Public Health. 2020;17(4):1257.

Page 29/34 
31. Schreinemachers P, Baliki G, Shrestha RM, Bhattarai DR, Gautam IP, Ghimire PL, Subedi BP, Brück T. Nudging children toward healthier food choices: An experiment combining school and home gardens. Global Food Security. 2020;26:100454.

32. Shrestha A, Schindler C, Odermatt P, Gerold J, Erismann S, Sharma S, Koju R, Utzinger J, Cissé G. Nutritional and health status of children 15 months after integrated school garden, nutrition, and water, sanitation and hygiene interventions: a cluster-randomised controlled trial in Nepal. BMC Public Health. 2020;20(1):1-19.

33. van den Berg A, Warren JL, Mclntosh A, Hoelscher D, Ory MG, Jovanovic C, Lopez M, Whittlesey L, Kirk A, Walton C. Impact of a gardening and physical activity intervention in Title 1 schools: The TGEG study. Childhood Obesity. 2020;16 Suppl 1:44-54.

34. Khan M, Bell R. Effects of a School Based Intervention on Children's Physical Activity and Healthy Eating: A Mixed-Methods Study. International Journal of Environmental Research and Public Health. 2019;16(22):4320.

35. Landry MJ, Markowitz AK, Asigbee FM, Gatto NM, Spruijt-Metz D, Davis JN. Cooking and gardening behaviors and improvements in dietary intake in Hispanic/Latino youth. Childhood Obesity. 2019;15(4):262-270.

36. Massarani FA, Citelli M, Canella DS, Koury JC. Healthy eating promoting in a Brazilian sports-oriented school: a pilot study. PeerJ. 2019;7:e7601.

37. Huys N, Cardon G, De Craemer M, Hermans N, Renard S, Roesbeke M, Stevens W, De Lepeleere S, Deforche B. Effect and process evaluation of a real-world school garden program on vegetable consumption and its determinants in primary schoolchildren. PloS One. 2019;14(3):e0214320.

38. Schreinemachers P, Ouedraogo MS, Diagbouga S, Thiombiano A, Kouamé SR, Sobgui CM, Chen H-P, Yang R-Y. Impact of school gardens and complementary nutrition education in Burkina Faso. Journal of Development Effectiveness. 2019;11(2):132-145.

39. Leuven JR, RUtenfrans AH, Dolfing AG, Leuven RS. School gardening increases knowledge of primary school children on edible plants and preference for vegetables. Food Science and Nutrition. 2018;6(7):1960-1967.

40. Taylor JC, Zidenberg-Cherr S, Linnell JD, Feenstra G, Scherr RE. Impact of a multicomponent, school-based nutrition intervention on students' lunchtime fruit and vegetable availability and intake: A pilot study evaluating the Shaping Healthy Choices Program. Journal of Hunger \& Environmental Nutrition. 2018;13(3):415-428.

41. Wells NM, Meyers BM, Todd LE, Henderson Jr CR, Barale K, Gaolach B, Ferenz G, Aitken M, Caroline CT, Pattison KO. The carry-over effects of school gardens on fruit and vegetable availability at home: A randomized controlled trial with low-income elementary schools. Preventive Medicine. 2018;112:152-159.

42. Gatto NM, Martinez LC, Spruijt-Metz D, Davis JN. LA sprouts randomized controlled nutrition, cooking and gardening programme reduces obesity and metabolic risk in Hispanic/Latino youth. Pediatric Obesity. 2017;12(1):28-37.

43. Lee RE, Parker NH, Soltero EG, Ledoux TA, Mama SK, McNeill L. Sustainability via Active Garden Education (SAGE): results from two feasibility pilot studies. BMC Public Health. 2017;17(1):1-11.

44. Davis JN, Martinez LC, Spruijt-Metz D, Gatto NM. LA Sprouts: A 12-week gardening, nutrition, and cooking randomized control trial improves determinants of dietary behaviors. Journal of Nutrition Education and Behavior. 2016;48(1):2-11. e11.

45. Duncan MJ, Eyre E, Bryant E, Clarke N, Birch S, Staples V, Sheffield D. The impact of a school-based gardening intervention on intentions and behaviour related to fruit and vegetable consumption in children. Journal of Health Psychology. 2015;20(6):765-773.

46. Sharma SV, Hedberg AM, Skala KA, Chuang R-J, Lewis T. Feasibility and acceptability of a gardening-based nutrition education program in preschoolers from low-income, minority populations. Journal of Early Childhood Research. 2015;13(1):93-110.

47. Spears-Lanoix EC, McKyer ELJ, Evans A, McIntosh WA, Ory M, Whittlesey L, Kirk A, Hoelscher DM, Warren JL. Using family-focused garden, nutrition, and physical activity programs to reduce childhood obesity: the texas! go! eat! grow! pilot study. Childhood Obesity. 2015;11(6):707714.

48. Wells NM, Myers BM, Todd LE, Barale K, Gaolach B, Ferenz G, Aitken M, Henderson CR, Tse C, Pattison KO. The effects of school gardens on children's science knowledge: a randomized controlled trial of low-income elementary schools. International Journal of Science Education 2015;37(17):2858-2878.

49. Yoder ABB, Liebhart JL, McCarty DJ, Meinen A, Schoeller D, Vargas C, LaRowe T. Farm to elementary school programming increases access to fruits and vegetables and increases their consumption among those with low intake. Journal of Nutrition Education and Behavior. 2014;46(5):341-349

50. Cotter J, Cotter MJ, Oliveira P, Cunha P, Polónia J. Salt intake in children 10-12 years old and its modification by active working practices in a school garden. Journal of Hypertension. 2013;31(10):1966-1971.

51. Gibbs L, Staiger PK, Johnson B, Block K, Macfarlane S, Gold L, Kulas J, Townsend M, Long C, Ukoumunne O. Expanding children's food experiences: the impact of a school-based kitchen garden program. Journal of Nutrition Education and Behavior. 2013;45(2):137-146.

52. Gatto NM, Ventura EE, Cook LT, Gyllenhammer LE, Davis JN. LA Sprouts: a garden-based nutrition intervention pilot program influences motivation and preferences for fruits and vegetables in Latino youth. Journal of the Academy of Nutrition and Dietetics. 2012;112(6):913-920. 
53. Jaenke RL, Collins CE, Morgan PJ, Lubans DR, Saunders KL, Warren JM. The impact of a school garden and cooking program on boys' and girls' fruit and vegetable preferences, taste rating, and intake. Health Education \& Behavior. 2012;39(2):131-141.

54. Davis JN, Ventura EE, Cook LT, Gyllenhammer LE, Gatto NM. LA Sprouts: a gardening, nutrition, and cooking intervention for Latino youth improves diet and reduces obesity. Journal of the American Dietetic Association. 2011;111(8):1224-1230.

55. Morgan PJ, Warren JM, Lubans DR, Saunders KL, Quick GI, Collins CE. The impact of nutrition education with and without a school garden on knowledge, vegetable intake and preferences and quality of school life among primary-school students. Public Health Nutrition. 2010;13(11):1931-1940.

56. Parmer SM, Salisbury-Glennon J, Shannon D, Struempler B. School gardens: an experiential learning approach for a nutrition education program to increase fruit and vegetable knowledge, preference, and consumption among second-grade students. Journal of Nutrition Education and Behavior. 2009;41(3):212-217.

57. Somerset S, Markwell K. Impact of a school-based food garden on attitudes and identification skills regarding vegetables and fruit: a 12-month intervention trial. Public Health Nutrition. 2009;12(2):214-221.

58. McAleese JD, Rankin LL. Garden-based nutrition education affects fruit and vegetable consumption in sixth-grade adolescents. Journal of the American Dietetic Association. 2007;107(4):662-665.

59. Bandura A, Freeman W, Lightsey R. Self-efficacy: The exercise of control. In.: Springer. 1999.

60. Schrader P, Lawless KA. The knowledge, attitudes, \& behaviors approach how to evaluate performance and learning in complex environments. Performance Improvement. 2004;43(9):8-15.

61. Contento IR, Manning AD, Shannon B. Research perspective on school-based nutrition education. Journal of Nutrition Education. 1992;24(5):247-260.

62. Berezowitz CK, Bontrager Yoder AB, Schoeller DA. School gardens enhance academic performance and dietary outcomes in children. Journal of School Health. 2015;85(8):508-518.

63. Falciglia GA, Couch SC, Gribble LS, Pabst SM, Frank R. Food neophobia in childhood affects dietary variety. Journal of the American Dietetic Association. 2000;100(12):1474-1481.

64. Cooke L, Wardle J, Gibson E. Relationship between parental report of food neophobia and everyday food consumption in 2-6-year-old children. Appetite. 2003;41(2):205-206.

65. Russell CG, Worsley A. Why don't they like that? And can I do anything about it? The nature and correlates of parents' attributions and selfefficacy beliefs about preschool children's food preferences. Appetite. 2013;66:34-43.

66. Juhl HJ, Poulsen CS. Antecedents and effects of consumer involvement in fish as a product group. Appetite. 2000;34(3):261-267.

67. Verbeke W, Vackier I. Individual determinants of fish consumption: application of the theory of planned behaviour. Appetite. 2005;44(1):67-82.

68. Morris J, Neustadter A, Zidenberg-Cherr S. First-grade gardeners more likely to taste vegetables. California Agriculture. 2001;55(1):43-46.

69. Wardle J, Cooke LJ, Gibson EL, Sapochnik M, Sheiham A, Lawson M. Increasing children's acceptance of vegetables; a randomized trial of parent-led exposure. Appetite. 2003;40(2):155-162.

70. Lakkakula A, Geaghan J, Zanovec M, Pierce S, Tuuri G. Repeated taste exposure increases liking for vegetables by low-income elementary school children. Appetite. 2010;55(2):226-231.

71. Scaglioni S, Salvioni M, Galimberti C. Influence of parental attitudes in the development of children eating behaviour. British Journal of Nutrition. 2008;99(S1):S22-S25.

72. Salvy S-J, de la Haye K, Bowker JC, Hermans RC. Influence of peers and friends on children's and adolescents' eating and activity behaviors. Physiology \& Behavior. 2012;106(3):369-378.

73. Santiago-Torres M, Adams AK, Carrel AL, LaRowe TL, Schoeller DA. Home food availability, parental dietary intake, and familial eating habits influence the diet quality of urban Hispanic children. Childhood Obesity. 2014;10(5):408-415.

74. Kristjansdottir AG, De Bourdeaudhuij I, Klepp K-I, Thorsdottir I. Children's and parents' perceptions of the determinants of children's fruit and vegetable intake in a low-intake population. Public Health Nutrition. 2009;12(8):1224-1233.

75. Fischer C, Brug J, Tak NI, Yngve A, te Velde SJ. Differences in fruit and vegetable intake and their determinants among 11-year-old schoolchildren between 2003 and 2009. International Journal of Behavioral Nutrition and Physical Activity. 2011;8(1):1-11.

76. Grosso G, Mistretta A, Turconi G, Cena H, Roggi C, Galvano F. Nutrition knowledge and other determinants of food intake and lifestyle habits in children and young adolescents living in a rural area of Sicily, South Italy. Public Health Nutrition. 2013;16(10):1827-1836.

77. Glanz K, Rimer BK, Viswanath K. Health behavior and health education: theory, research, and practice. John Wiley \& Sons. 2008.

78. Smelser NJ, Baltes PB. International encyclopedia of the social \& behavioral sciences. Amsterdam: Elsevier; 2001.

79. Frymier AB, Nadler MK. Persuasion: Integrating theory, research, and practice. Recording for the Blind \& Dyslexic. 2007.

80. Rogers R, Prentice-Dunn S, Gochman D. Handbook of health behavior research 1: Personal and social determinants. New York, NY, US: Plenum Press; 1997. p. 113-132. 
81. Willows ND, Veugelers P, Raine K, Kuhle S. Prevalence and sociodemographic risk factors related to household food security in Aboriginal peoples in Canada. Public Health Nutrition. 2009;12(8):1150-1156.

82. Findlay LC, Langlois KA, Kohen DE. Hunger among Inuit children in Canada. International Journal of Circumpolar Health. 2013;72(1):20324.

83. Birch LL. The relationship between children's food preferences and those of their parents. Journal of Nutrition Education. 1980;12(1):14-18.

84. Birch LL, Fisher JO. Development of eating behaviours among children and adolescents. Pediatrics. 1998;101(suppl 2):539-549.

85. Dubowitz T, Heron M, Bird CE, Lurie N, Finch BK, Basurto-Dávila R, Hale L, Escarce JJ. Neighborhood socioeconomic status and fruit and vegetable intake among whites, blacks, and Mexican Americans in the United States. The American Journal of Clinical Nutrition. 2008;87(6):1883-1891.

86. Pollard J, Kirk SL, Cade JE. Factors affecting food choice in relation to fruit and vegetable intake: a review. Nutrition Research Reviews. 2002;15(2):373-387.

87. Jago R, Baranowski T, Baranowski JC. Fruit and vegetable availability: a micro environmental mediating variable? Public Health Nutrition. 2007;10(7):681-689.

88. Murimi MW, Moyeda-Carabaza AF, Nguyen B, Saha S, Amin R, Njike V. Factors that contribute to effective nutrition education interventions in children: a systematic review. Nutrition Reviews. 2018;76(8):553-580.

89. Jeong J, Pitchik HO, Fink G: Short-term, medium-term and long-term effects of early parenting interventions in low-and middle-income countries: a systematic review. BMJ Global Health. 2021;6(3):e004067.

90. Davis JN, Spaniol MR, Somerset S. Sustenance and sustainability: maximizing the impact of school gardens on health outcomes. Public Health Nutrition 2015;18(13):2358-2367.

91. Edwards R, Alldred P. A typology of parental involvement in education centring on children and young people: Negotiating familialisation, institutionalisation and individualisation. British Journal of Sociology of Education. 2000;21(3):435-455.

92. Richardson SA. Principals' perceptions of parental involvement in the" big 8" urban districts of Ohio. Research in the Schools. 2009;16(1):1.

93. Sanders MG, Sheldon SB. Principals matter: A guide to school, family, and community partnerships. Corwin Press; 2009.

94. Epstein JL, Sanders MG, Sheldon SB, Simon BS, Salinas KC, Jansorn NR, Van Voorhis FL, Martin CS, Thomas BG, Greenfeld MD. School, family, and community partnerships: Your handbook for action. Corwin Press; 2018.

95. Bandura A, Walters RH. Social learning theory, vol. 1. Englewood cliffs Prentice Hall; 1977.

96. Bronfenbrenner U. The ecology of human development: Experiments by nature and design. Harvard university press; 1979.

97. Hedenbro M, Rydelius PA. Children's abilities to communicate with both parents in infancy were related to their social competence at the age of 15. Acta Paediatrica. 2019;108(1):118-123.

98. Lamb ME, Bornstein MH. Development in infancy: An introduction: Crown Publishing Group/Random House; 1987.

99. Đurišić M, Bunijevac M. Parental involvement as an important factor for successful education. Center for Educational Policy Studies Journal. 2017;7(3):137-153.

100. Centres for Disease Control and Prevention. Strategies for Involving Parents in School Health. Atlanta, GA: U.S Department of Health and Human Services; 2012.

101. Zhao J-G. Combination of multiple databases is necessary for a valid systematic review. International orthopaedics. 2014;38(12):2639-2639.

102. Haidich A-B. Meta-analysis in medical research. Hippokratia. 2010;14 Suppl 1:29.

103. Rockoff JE. The impact of individual teachers on student achievement: Evidence from panel data. American Economic Review. 2004;94(2):247252.

\section{Figures}




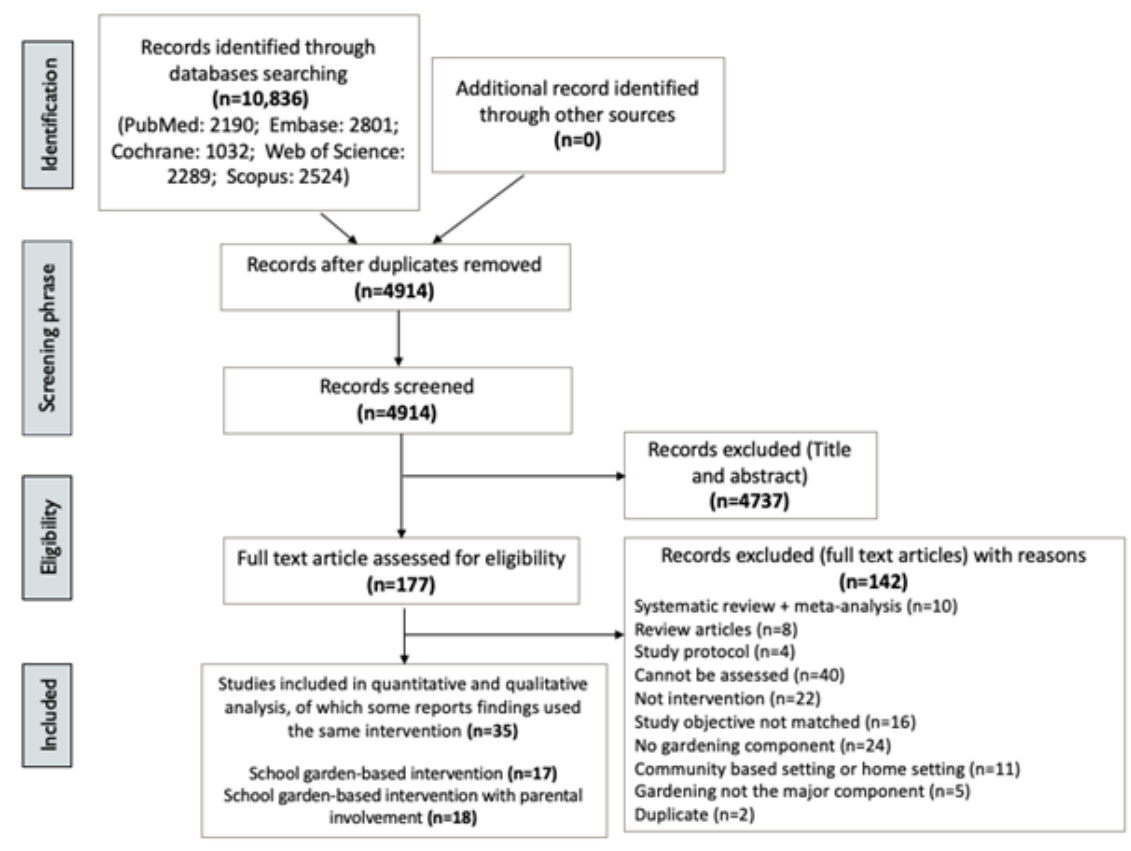

Figure 1

Flowchart of identification and selection of studies in accordance with PRISMA guidelines

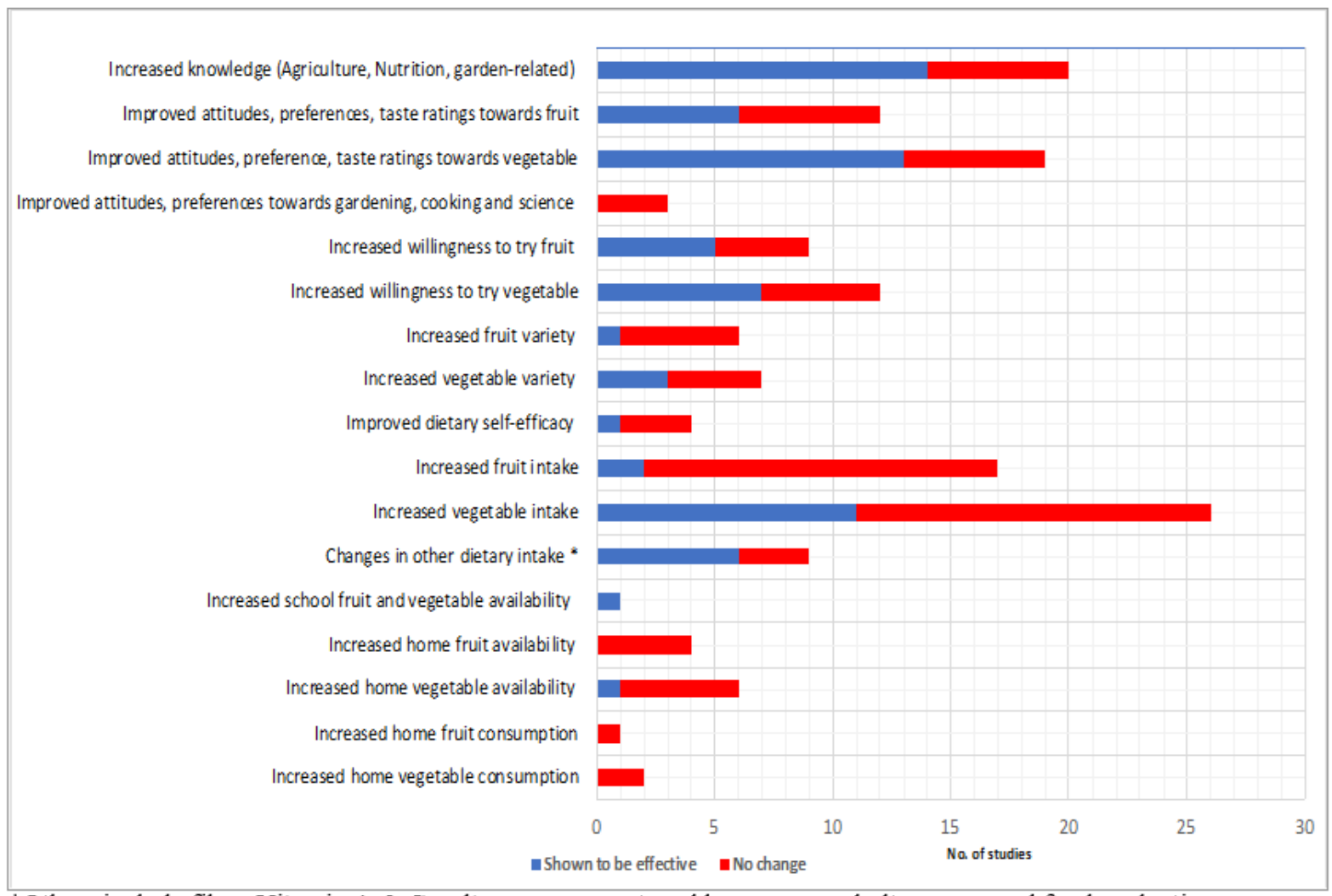

* Others include fibre, Vitamin A \& C, salts, sugary sweetened beverages and ultra-processed foods reduction.

\section{Figure 2}

Findings from the included SGBP intervention $(n=35)$ 


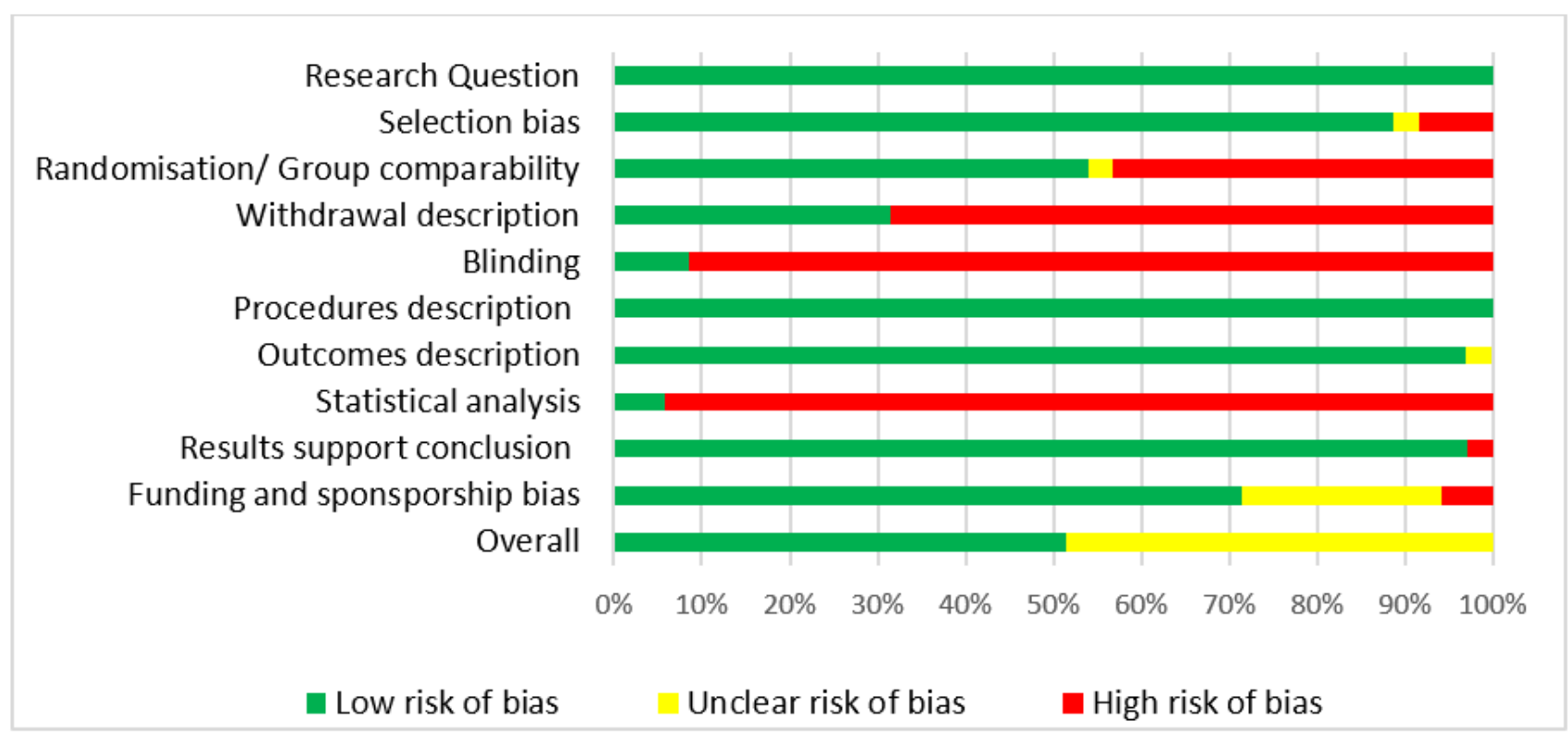

Figure 3

Quality rating of included studies using the Quality Criteria Checklist from Academy of Nutrition and Dietetics

\section{Supplementary Files}

This is a list of supplementary files associated with this preprint. Click to download.

- SupplementaryMaterials.docx 